Preprint de "On strategy-proofness and symmetric single-peakedness," de Jordi Massó i Inés Moreno de Barreda. Games and Economic Behavior 72, 467-484 (2011). Lliurat a Elsevier el novembre de 2010. 


\title{
On Strategy-proofness and Symmetric Single-peakedness
}

\author{
Jordi Massó $^{* \dagger} \quad$ Inés Moreno de Barreda
}

November 2010

\begin{abstract}
We characterize the class of strategy-proof social choice functions on the domain of symmetric single-peaked preferences. This class is strictly larger than the set of generalized median voter schemes (the class of strategy-proof and tops-only social choice functions on the domain of single-peaked preferences characterized by Moulin, 1980) since, under the domain of symmetric single-peaked preferences, generalized median voter schemes can be disturbed by discontinuity points and remain strategy-proof on the smaller domain. Our result identifies the specific nature of these discontinuities which allow to design non-onto social choice functions to deal with feasibility constraints.
\end{abstract}

JEL Classification: D7.

Keywords: Strategy-proofness, Single-peaked Preferences, Median Voter, Feasibility Constraints.

${ }^{*}$ Corresponding author. Telephone number: +34 935812370. Fax number: +34 935812461.

†'Departament d'Economia i d'Història Econòmica and CODE, Universitat Autònoma de Barcelona, 08193 Bellaterra (Barcelona), Spain; e-mail: jordi.masso@uab.es

${ }^{\ddagger}$ Department of Economics and STICERD, London School of Economics, Houghton Street, London WC2A 2AE, United Kingdom; e-mail: i.moreno-de-barreda@lse.ac.uk 


\section{Introduction}

Consider a society with $n$ agents who have to collectively choose one alternative from a given set of social alternatives. Assume that this set is endowed with a natural strict order because alternatives have a common characteristic according to which pairs of alternatives can be compared in an objective way. For instance, the set of alternatives may consist of physical locations (a public facility on a road or street), properties of a political project in terms of its left-right characteristics, the expenditure level on a public good, indexes reflecting the quality of a product, feasible temperatures in a room, and so on. ${ }^{1}$ In all these cases, and in many others, this linear order structure permits to identify the set of alternatives with a subset of the real line. Agents have (potentially different) preferences on the set of alternatives. Black (1948) is the first to suggest that, given the linear order on the set of alternatives, agents' preferences ought to be singlepeaked. The preference of an agent is single-peaked if there exists an alternative (called the top) which is strictly preferred to any other alternative and on each side of the top the preference is strictly monotonic, increasing on its left and decreasing on its right. ${ }^{2}$

Society would like to select an alternative according to agents' preferences. But since they constitute private information, agents have to be asked about them. A social choice function on a domain of preferences requires each agent to report a preference and associates an alternative with the reported preference profile. Hence, a social choice function on a Cartesian product domain induces an (ordinal) direct revelation game where each agent's set of strategies is his set of possible preferences. A social choice function is strategy-proof if no agent has ever incentives to strategically misrepresent his preference; in other words, truth-telling is a (weakly) dominant strategy in the direct revelation game induced by the social choice function.

Moulin (1980) characterizes the class of strategy-proof and tops-only social choice functions on the domain of single-peaked preferences as the set of generalized median voter schemes. ${ }^{3}$ A generalized median voter scheme is, in general, a non-anonymous ex-

\footnotetext{
${ }^{1}$ There is an extensive literature studying collective choice problems where the set of social alternatives is a linearly ordered set. See Moulin (1980), for instance. This class of problems also plays a fundamental role in Sprumont (1995) and Barberà (2001, 2010), three excellent surveys on strategyproofness.

${ }^{2}$ The set of single-peaked preferences is extremely large and rich; for instance, for each alternative there are many single-peaked preferences that have as top this alternative. Moreover, no a priori restriction is imposed on how pairs of alternatives lying in different sides of the top are ordered. Ballester and Haeringer (2010) identify two properties that are both necessary and sufficient to characterize the domain of single-peaked preference profiles.

${ }^{3} \mathrm{~A}$ social choice function is tops-only if the chosen alternative only depends on the profile of tops.
} 
tension of the median voter. It can be interpreted as a particular way of distributing the power to influence the social outcome among all coalitions of agents. In addition, Moulin (1980) also identifies the two nested subclasses of strategy-proof, tops-only and anonymous social choice functions, and strategy-proof, tops-only, anonymous and efficient social choice functions. ${ }^{4}$ The ranges of all functions in Moulin (1980)'s characterizations are closed intervals. This implies that if some alternatives were banned or infeasible, either the social choice function would have to request from the agents more information than just their tops, or there would be a single-peaked preference profile and an agent with incentives to misreport his preferences.

In many applications however, the domain of preferences can be restricted even further because the linear order structure of the set of alternatives conveys to agents' preferences more than just an ordinal content. Often, an agent's preference on the set of alternatives is responsive also to the notion of distance, embedding to the preference its corresponding property of symmetry. A single-peaked preference is symmetric if the following additional condition holds: an alternative is strictly preferred to another one if and only if the former is strictly closer to the top. If an indifference class contains two alternatives then both are located in opposite sides of the top and are at the same distance of the top. ${ }^{5}$

To restrict further the domain of a social choice function is equivalent to shrink the set of agents' strategies in its induced direct revelation game. Thus, strategies that were dominant remain dominant while strategies that were not dominant in the larger domain may become dominant after the domain reduction. Therefore, two important facts hold. First, any strategy-proof social choice function on a domain remains strategyproof on all of its subdomains. Second, a manipulable social choice function on a domain may become strategy-proof in a smaller subdomain. ${ }^{6}$ Hence, we ask whether the set

Tops-only social choice functions are especially simple in terms of the amount of information they require about individual preferences. Ching (1997) gives an alternative description and several axiomatic characterizations of generalized median voter schemes; in particular, Ching (1997) shows that in Moulin (1980)'s characterization tops-onlyness can be replaced by continuity.

\footnotetext{
${ }^{4} \mathrm{~A}$ social choice function is anonymous if it is independent of the identities of the agents; it is efficient if it always selects a Pareto optimal alternative.

${ }^{5}$ The notion of symmetric single-peakedness has already been considered in the context of strategyproofness; for example in Border and Jordan (1983), Peters et al. (1992), Klaus et al. (1998), Ehlers (2002), Nisan (2007), Kar and Kibris (2008), and Klaus and Bochet (2010). It has also been considered in the context of Political Economy to model voters' preferences over policies identified with an interval; for example in McKelvey and Ordeshook (1993) and Krehbiel (2006).

${ }^{6}$ Observe two things. First, this is just a possibility. For instance, for the case where the set of social alternatives is the family of all subsets of a given set of candidates Barberà et al. (1991) show
} 
of strategy-proof and tops-only social choice functions on the domain of single-peaked preferences, identified by Moulin (1980) as the class of generalized median voter schemes, becomes larger when the domain of preferences where we want the social choice functions to operate is the subdomain of symmetric single-peaked preferences. We answer this question affirmatively by completely identifying the larger class of functions that emerge after restricting further the domain.

The new class of social choice functions can be described as generalized median voter schemes disturbed by discontinuity jumps. A social choice function $f$ in the class coincides with a generalized median voter scheme except that at some (countable number of) discontinuity jumps (for instance, an interval $(a, b)$ with midpoint $d$ ), instead of taking the value prescribed by the generalized median voter scheme, $f$ takes the constant value $a$ at $[a, d)$, either the value $a$ or $b$ at $d$, and the constant value $b$ at $(d, b]$. Our description of the class makes precise that the choice of either $a$ or $b$ at any of those profiles where the generalized median voter scheme would choose $d$ must be monotonic in order to preserve strategy-proofness of the social choice function.

We want to stress the importance for applications of admitting discontinuous social choice functions that are non-onto because they have a disconnected range, and this range can in fact be any closed subset of alternatives. Besides, this range can be chosen beforehand. Non-onto social choice functions are indispensable for the design of social choice functions that require that some subsets of alternatives are never chosen due to feasibility constraints. For instance when the range of the function has to be finite, or not all locations for a public facility are possible, or the set of indexes reflecting the quality of a product must be disconnected, or the thermostat controlling for the temperature in a room can not take all values, and so on. In all these cases, and in many others, discontinuities can not be regarded as pathological features of social choice functions but rather as indispensable requirements to deal with constraints on the set of feasible alternatives to be chosen. ${ }^{7}$

that voting by committees is the class of strategy-proof and onto social choice functions on both, the domain of separable preferences as well as on the subdomain of additive preferences, although the set of additive preferences is strictly smaller than the set of separable preferences. No new strategy-proof social choice function appears after the domain reduction in this case. Second, given a tops-only social choice function on the domain of single-peaked preferences, the set of agents' strategies in its induced direct revelation game is smaller when single-peaked preferences are further restricted to be symmetric because the fact that the rule is constant (by tops-onlyness) in a large subset of profiles is unrelated with the fact that the set of preferences that agents may use to evaluate the outcomes of the social choice function is smaller.

${ }^{7}$ Barberà et al. (1997, 2005) and Barberà et al. (1998) identify subclasses of strategy-proof social choice functions that are able to deal with constrained sets of alternatives in different environments. 
There is a large literature studying strategy-proofness on domains related to singlepeakedness. Border and Jordan (1983) extend Moulin (1980)'s results to multi-dimensional environments. One of the domains they consider is the set of quadratic separable preferences that coincides with the domain of symmetric single-peaked preferences when the number of dimensions is equal to one. However, Border and Jordan (1983) only consider social choice functions that respect unanimity (i.e., if all agents have the same top then the common top should be chosen). Hence, all their results apply only to social choice functions whose ranges coincide with the set of alternatives. In particular, they show that for the one-dimensional case strategy-proof social choice functions that respect unanimity on the domain of symmetric single-peaked preferences are uncompromising, ${ }^{8}$ and the partial converse that all uncompromising social choice functions are strategy-proof; moreover, all uncompromising social choice functions on this domain are continuous. Nehring and Puppe (2007a, 2007b) study strategy-proofness in rich domains satisfying a general notion of single-peakedness based on abstract betweenness relations. However, their richness condition explicitly excludes as an admitted domain the set of symmetric single-peaked preferences since it requires that for any triple of alternatives $(y, x, z)$ with $y$ not being between $x$ and $z$ there must exist a preference relation in the domain with top on $x$ such that $z$ is strictly preferred to $y$. Thus, their results and ours are logically unrelated.

Our result and its proof are closely related to the following papers. Theorem 1 partly retains the structure of Moulin (1980)'s characterization of strategy-proof and tops-only social choice functions under the single-peaked domain of preferences. Our result in Theorem 1 says that strategy-proof social choice functions on the symmetric single-peaked domain that are manipulable on the larger single-peaked domain consists of generalized median voter schemes that are perturbed by specific discontinuities. Our result is also related to Theorem 3 in Barberà and Jackson (1994) characterizing all strategy-proof social choice functions on the domain of single-peaked preferences. Their characterization includes social choice functions whose range is not an interval; however, the characterization is open because it relies on a family of tie-breaking rules (used to select between the two extremes of the discontinuity jumps) that are not fully described. Our characterization is closed because it explicitly describes the exact family of admissible tie-breaking rules needed to preserve strategy-proofness. Yet, we are able to provide this closed description because our domain contains only symmetric preferences. The

\footnotetext{
${ }^{8} \mathrm{~A}$ social choice function is uncompromising if an agent's top lies to the right (respectively, left) of the chosen alternative $x$, then any change in the top which leaves it to the right (respectively, left) of $x$ will not affect the choice.
} 
proof of our result relays at some point on Berga and Serizawa (2000)'s characterization of all strategy-proof and onto social choice functions on a minimally rich domain as the class of generalized median voter schemes; ${ }^{9}$ we use their result in the easier case when the given strategy-proof social choice function is continuous. In addition, our proof is substantially simpler than it would have been if we were not able to use Barberà et al. (2010) result identifying conditions of preference domains under which (individual) strategy-proofness is equivalent to group strategy-proofness. Their result allows us to avoid many steps of individual changes of preferences by instead moving simultaneously the preferences of all members of a given coalition.

The paper is organized as follows. In Section 2 we present preliminary notations and the most basic definitions. In Section 3 we state some previous results and give the main definitions and intuitions in order to understand why and how the class of generalized median voter schemes has to be enlarged in order to identify the full class of strategy-proof social choice functions on the domain of symmetric single-peaked preferences. In Section 4 we state and prove our main result characterizing the complete class of strategy-proof social choice functions on the domain of symmetric single-peaked preferences (Theorem 1). After presenting some preliminaries of the proof in Subsection 4.2, we prove Theorem 1 in Subsection 4.3. In Section 5 we first state as corollaries of Theorem 1 the corresponding characterizations under strategy-proofness and anonymity (Corollary 1) and under strategy-proofness, anonymity and efficiency (Corollary 2). We then argue about the importance for applications of allowing for non-onto social choice functions which were ruled out by the combination of strategy-proofness and tops-onlyness in Moulin (1980)'s characterization under single-peaked preferences and state Corollary 3 characterizing all strategy-proof social choice functions that are efficient relative to a given closed set of feasible alternatives. We finish with the remark that, as the consequence of the main result in Barberà et al. (2010), the four statements hold if we replace in them strategy-proofness by group strategy-proofness.

\footnotetext{
${ }^{9} \mathrm{~A}$ domain is minimally rich if (i) it is a subset of the single-peaked domain, (ii) for each alternative $x$ there is a preference relation in the domain with top at $x$, and (iii) for any pair of alternatives $x$ and $y(x \neq y)$ there is a preference in the domain that strictly orders $x$ and $y$ and whose top lies between $x$ and $y$. Obviously, the set of symmetric single-peaked preferences is a minimally rich domain.
} 


\section{Preliminary notations and definitions}

Let $N=\{1, \ldots, n\}$ be the set of agents of a society that has to choose an alternative $x$ from the interval $[0,1] .{ }^{10}$ Subset of agents will be denoted by capital letters (like $S$ ) and their cardinalities by their corresponding small letters (like $s$ ). The preference of each agent $i \in N$ on the set of alternatives $[0,1]$ is a complete, reflexive, and transitive binary relation (a complete preorder) $R_{i}$ on $[0,1]$. Let $\mathcal{R}$ be the set of complete preorders on $[0,1]$. A preference profile $R=\left(R_{1}, \ldots, R_{n}\right) \in \mathcal{R}^{n}$ is a $n$-tuple of preferences. To emphasize the role of agent $i$ or subset of agents $T$, a preference profile $R$ will be represented by $\left(R_{i}, R_{-i}\right)$ or $\left(R_{T}, R_{-T}\right)$, respectively. As usual, let $P_{i}$ and $I_{i}$ denote the strict and indifference preference relations induced by $R_{i}$, respectively. Given $R_{i} \in \mathcal{R}$, the top of $R_{i}$ (if any) is the unique alternative $t\left(R_{i}\right)$ that is strictly preferred to any other alternative; i.e., $t\left(R_{i}\right) P_{i} x$ for all $x \in[0,1] \backslash\left\{t\left(R_{i}\right)\right\}$.

Given a subset of preferences $\mathcal{S} \subseteq \mathcal{R}$, a social choice function (SCF from now on) $f$ on $\mathcal{S}$ is a function $f: \mathcal{S}^{n} \rightarrow[0,1]$ selecting an alternative for each preference profile in $\mathcal{S}^{n}$. We will refer to this Cartesian product set $\mathcal{S}^{n}$ (or to the set $\mathcal{S}$ itself) as the domain of preferences. Given a SCF $f: \mathcal{S}^{n} \rightarrow[0,1]$, denote its range by $r_{f}$; i.e., $r_{f}=\left\{x \in[0,1] \mid\right.$ there exists $R \in \mathcal{S}^{n}$ such that $\left.f(R)=x\right\}$.

We will be interested in SCFs that induce truth-telling as a (weakly) dominant strategy in their associated (ordinal) direct revelation game.

Definition 1 A SCF $f: \mathcal{S}^{n} \rightarrow[0,1]$ is strategy-proof if for all $R \in \mathcal{S}^{n}$, all $i \in N$, and all $R_{i}^{\prime} \in \mathcal{S}$

$$
f\left(R_{i}, R_{-i}\right) R_{i} f\left(R_{i}^{\prime}, R_{-i}\right)
$$

If $f\left(R_{i}^{\prime}, R_{-i}\right) P_{i} f(R)$ we say that $i$ manipulates $f$ at $R$ via $R_{i}^{\prime}$. A SCF $f: \mathcal{S}^{n} \rightarrow[0,1]$ is group strategy-proof if for all $R \in \mathcal{S}^{n}$, all $T \subseteq N$, and all $R_{T}^{\prime} \in \mathcal{S}^{t}$ with $R_{i}^{\prime} \neq R_{i}$ for all $i \in T$,

$$
f\left(R_{T}, R_{-T}\right) R_{i} f\left(R_{T}^{\prime}, R_{-T}\right)
$$

for some $i \in T$.

If $f\left(R_{T}^{\prime}, R_{-T}\right) P_{i} f(R)$ for all $i \in T$ we say that $T$ manipulates $f$ at $R$ via $R_{T}^{\prime}$.

We will also consider other properties of SCFs. A SCF $f: \mathcal{S}^{n} \rightarrow[0,1]$ is anonymous if it is invariant with respect to the agents' names; namely, for all one-to-one mappings $\sigma: N \rightarrow N$ and all $R \in \mathcal{S}^{n}, f\left(R_{1}, \ldots, R_{n}\right)=f\left(R_{\sigma(1)}, \ldots, R_{\sigma(n)}\right)$. A SCF $f: \mathcal{S}^{n} \rightarrow[0,1]$

\footnotetext{
${ }^{10}$ Our results also hold for any linearly ordered metric space of alternatives. In particular, for any set of alternatives which is a closed interval of real numbers (as well as for the set $\mathbb{R} \cup\{-\infty,+\infty\}$ ).
} 
is efficient if for all $R \in \mathcal{S}^{n}$, there is no $z \in[0,1]$ such that, for all $i \in N, z R_{i} f(R)$ and $z P_{j} f(R)$ for some $j \in N .{ }^{11}$ A SCF $f: \mathcal{S}^{n} \rightarrow[0,1]$ is unanimous if for all $R \in \mathcal{S}^{n}$ such that $t\left(R_{i}\right)=x$ for all $i \in N, f(R)=x$. A SCF $f: \mathcal{S}^{n} \rightarrow[0,1]$ is onto if for all $x \in[0,1]$ there is $R \in \mathcal{S}^{n}$ such that $f(R)=x$ (i.e., $r_{f}=[0,1]$ ). A SCF $f: \mathcal{S}^{n} \rightarrow[0,1]$ is tops-only if for all $R, R^{\prime} \in \mathcal{S}^{n}$ such that $t\left(R_{i}\right)=t\left(R_{i}^{\prime}\right)$ for all $i \in N, f(R)=f\left(R^{\prime}\right)$. Let $\mathcal{S} \subseteq \mathcal{R}$ be any subset of preferences with the property that for each $x \in[0,1]$ there exists at least one preference $R_{i} \in \mathcal{S}$ such that $t\left(R_{i}\right)=x$. Then, $\mathcal{S}^{n}$ is called a rich domain and with some abuse of notation, given a tops-only SCF $f: \mathcal{S}^{n} \rightarrow[0,1]$ we will refer to it by its corresponding voting scheme $f:[0,1]^{n} \rightarrow[0,1]$.

In many applications, a linear order structure on the set of alternatives naturally induces a domain restriction in which there always exists a top, and at each of the sides of the top the preference is strictly monotonic.

Definition 2 A preference $R_{i} \in \mathcal{R}$ is single-peaked if:

(1) there exists the top $t\left(R_{i}\right)$ of $R_{i}$, and

(2) for all $x, y \in[0,1]$ such that $y<x \leq t\left(R_{i}\right)$ or $t\left(R_{i}\right) \leq x<y, x P_{i} y$.

Let $\mathcal{S P}$ be the set of single-peaked preferences on $[0,1]$. Observe that, given a singlepeaked preference $R_{i} \in \mathcal{S P}, y P_{i} x$ may hold even if $\left|t\left(R_{i}\right)-x\right|<\left|t\left(R_{i}\right)-y\right|$; but then, $x$ and $y$ are necessarily located in different sides of the top $t\left(R_{i}\right)$. Often, the linear order structure of the set of alternatives and a distance conveys to the preference a symmetric property around the top (coming for instance, from a location interpretation of the set of alternatives) that naturally induces the restriction that preferences respond to the distance as follows.

Definition 3 A preference $R_{i} \in \mathcal{R}$ is symmetric single-peaked if:

(1) there exists the top $t\left(R_{i}\right)$ of $R_{i}$, and

(2) for all $x, y \in[0,1], x P_{i} y$ if and only if $\left|t\left(R_{i}\right)-x\right|<\left|t\left(R_{i}\right)-y\right|$.

Obviously, a symmetric single-peaked preference is single-peaked. Let $\mathcal{S S P}$ be the set of symmetric single-peaked preferences on $[0,1]$. Given any alternative $x \in[0,1]$, there is a unique symmetric single-peaked preference $R_{i}$ with its top $t\left(R_{i}\right)=x(\mathcal{S S P}$ is a rich domain). Hence, there is a one-to-one mapping between the set of symmetric single-peaked preferences $\mathcal{S S P}$ and the set of alternatives $[0,1]$. Thus, we will use $t_{i} \in[0,1]$ to identify the (unique) $R_{i} \in \mathcal{S S P}$ such that $t\left(R_{i}\right)=t_{i}$ and $t=\left(t_{1}, \ldots, t_{n}\right)$ to denote the corresponding symmetric single-peaked preference profile $R=\left(R_{1}, \ldots, R_{n}\right)$

\footnotetext{
${ }^{11}$ In Subsection 5.2 we will define the notion of efficiency relative to a subset of alternatives $A \subseteq r_{f}$ by replacing the above condition "there is no $z \in[0,1]$ " by "there is no $z \in A$ ".
} 
such that $t\left(R_{i}\right)=t_{i}$ for all $i \in N$. Note that, by this one-to-one identification, any SCF $f: \mathcal{S S P}^{n} \rightarrow[0,1]$ is tops-only. Thus, we will also denote a SCF $f: \mathcal{S S P}^{n} \rightarrow[0,1]$ by its corresponding voting scheme $f:[0,1]^{n} \rightarrow[0,1]$. Following Berga and Serizawa (2000) a subset $\mathcal{S} \subseteq \mathcal{S P}$ is a minimally rich domain if it is rich and for any pair of alternatives $x, y \in[0,1], x \neq y$, there exists $R_{i} \in \mathcal{S}$ such that $x P_{i} y$ and $t\left(R_{i}\right) \in$ $(\min \{x, y\}, \max \{x, y\})$. Observe that $\mathcal{S} \mathcal{S} \mathcal{P}^{n}$ is a minimally rich domain.

\section{Previous results and main intuition}

\subsection{Previous results}

Moulin (1980) characterizes the family of strategy-proof and tops-only SCFs on the domain of single-peaked preferences as well as its anonymous subfamily. ${ }^{12}$ The two characterizations are useful to develop helpful intuitions to understand our characterization of strategy-proof SCFs (and its anonymous subfamily) on the domain of symmetric single-peaked preferences. To state them, we need to define the median of an odd set of numbers and the notion of a monotonic family of fixed ballots. Given a set of odd real numbers $\left\{x_{1}, \ldots, x_{K}\right\}$, define its median as $\operatorname{med}\left\{x_{1}, \ldots, x_{K}\right\}=y$, where $y$ is such that $\#\left\{1 \leq k \leq K \mid x_{k} \leq y\right\} \geq \frac{K}{2}$ and $\#\left\{1 \leq k \leq K \mid x_{k} \geq y\right\} \geq \frac{K}{2}$; observe that since $K$ is odd the median belongs to the set $\left\{x_{1}, \ldots, x_{K}\right\}$ and it is unique. A collection $\left\{p_{S}\right\}_{S \in 2^{N}}$ is a monotonic family of fixed ballots if $p_{S} \in[0,1]$ for all $S \in 2^{N}$ and $T \subset Q$ implies $p_{Q} \leq p_{T}$.

Proposition 1 (Moulin, 1980) ASCF $f: \mathcal{S P}^{n} \rightarrow[0,1]$ is strategy-proof, tops-only and anonymous if and only if there exist $n+1$ fixed ballots $0 \leq p_{n} \leq \ldots \leq p_{0} \leq 0$ such that for all $R \in \mathcal{S P}^{n}$,

$$
f(R)=\operatorname{med}\left\{t\left(R_{1}\right), \ldots, t\left(R_{n}\right), p_{n}, \ldots, p_{0}\right\} .
$$

Proposition 2 (Moulin, 1980) ASCF $f: \mathcal{S P}^{n} \rightarrow[0,1]$ is strategy-proof and tops-only if and only if there exists a monotonic family of fixed ballots $\left\{p_{S}\right\}_{S \in 2^{N}}$ such that for all $R \in \mathcal{S} \mathcal{P}^{n}$

$$
f(R)=\min _{S \in 2^{N}} \max _{i \in S}\left\{t\left(R_{i}\right), p_{S}\right\} .
$$

The SCFs identified in Propositions 1 and 2 are called median voter schemes and generalized median voter schemes, respectively. A simple way of interpreting them is as

\footnotetext{
${ }^{12}$ Moulin (1980) also characterizes the subfamily of strategy-proof, tops-only, anonymous and efficient SCFs on the domain of single-peaked preferences. See Corollary 2 in Section 5 for the characterization of the same class of SCFs on the domain of symmetric single-peaked preferences.
} 
follows. Each generalized median voting scheme (and its associated monotonic family of fixed ballots) can be understood as a particular way of distributing the power among coalitions to influence the social choice. To see that, take an arbitrary coalition $S$ and its fixed ballot $p_{S}$. Then, coalition $S$ can make sure that, by all of its members reporting a top alternative below $p_{S}$, the social choice will be at most $p_{S}$, independently of the reported top alternatives of the members of the complementary coalition. ${ }^{13}$ An alternative way of describing this distribution of power among coalitions is as follows. Fix a monotonic family of fixed ballots $\left\{p_{S}\right\}_{S \in 2^{N}}$ (i.e., a generalized median voter scheme) and take a vector of tops $\left(t\left(R_{1}\right), \ldots, t\left(R_{n}\right)\right)$. Start at the left extreme of the interval and push the outcome to the right until it reaches an alternative $x$ for which the following two things happen simultaneously: (i) there exists a coalition of agents $S$ such that all its members have reported a top alternative below or equal to $x$ (i.e., $t\left(R_{i}\right) \leq x$ for all $i \in S$ ) and (ii) the fixed ballot $p_{S}$ associated to $S$ is located also below $x$ (i.e., $\left.p_{S} \leq x\right)$. Median voter schemes are the anonymous subclass of generalized median voter schemes. Hence, the fixed ballots of any two coalitions with the same cardinality of any anonymous generalized median voter scheme are equal. From a monotonic family of fixed ballots $\left\{p_{S}\right\}_{S \in 2^{N}}$ associated to an anonymous generalized median voter scheme $f$ we can identify the $n+1$ ballots $p_{n} \leq p_{n-1} \leq \ldots \leq p_{0}$ needed to describe $f$ as a median voter scheme as follows: for each $0 \leq s \leq n, p_{s}=p_{S}$ for all $S \in 2^{N}$ such that $\# S=s$. Moreover, if $n$ is odd the (ordinary) median voter is obtained by choosing $p_{n}=\ldots=p_{\frac{n+1}{2}}=0$ and $p_{\frac{n+1}{2}-1}=\ldots=p_{0}=1$ since for any $R \in \mathcal{S P}^{n}$,

$$
\begin{aligned}
\operatorname{med}\left\{t\left(R_{1}\right), \ldots, t\left(R_{n}\right), p_{n}, \ldots, p_{0}\right\} & =\operatorname{med}\{t\left(R_{1}\right), \ldots, t\left(R_{n}\right), \underbrace{0, \ldots, 0}_{\frac{n+1}{2}-\text { times }}, \underbrace{1, \ldots, 1}_{\frac{n+1}{2}-\text { times }}\} \\
& =\operatorname{med}\left\{t\left(R_{1}\right), \ldots, t\left(R_{n}\right)\right\} .
\end{aligned}
$$

Finally, the SCF $f$ where agent $j \in N$ is the dictator (i.e., for all $R \in \mathcal{S P}^{n}, f(R)=$ $\left.t\left(R_{j}\right)\right)$ can be described as a generalized median voter scheme by setting $p_{T}=0$ for all $T \subset N$ such that $j \in T$ and $p_{S}=1$ for all $S \subset N$ such that $j \notin S$. Then, for any $R \in \mathcal{S P}^{n}, \max \left\{t\left(R_{j}\right), p_{\{j\}}\right\}=t\left(R_{j}\right)$, for any $T \subset N$ such that $j \in T, t\left(R_{j}\right) \leq$ $\max _{i \in T}\left\{t\left(R_{i}\right), p_{T}\right\}$, and for any $S \subset N$ such that $j \notin S, \max _{i \in S}\left\{t\left(R_{i}\right), p_{S}\right\}=1$. Thus, $\min _{S^{\prime} \in 2^{N}} \max _{i^{\prime} \in S^{\prime}}\left\{t\left(R_{i^{\prime}}\right), p_{S^{\prime}}\right\}=t\left(R_{j}\right)$.

Moulin (1980) also shows that the class of group strategy-proof and tops-only SCFs on the domain of single-peaked preferences coincides with the set of generalized median

\footnotetext{
${ }^{13}$ See Barberà et al. (1997) for a similar interpretation for the case of a finite number of ordered alternatives.
} 
voter schemes. From the main result in Barberà et al. (2010) we can conclude that any strategy-proof SCF on the domain of symmetric single-peaked preferences is group strategy-proof as well. Since we will later use this fact we state it here as a remark. ${ }^{14}$

Remark 1 (Barberà et al., 2010) Let $f: \mathcal{S S P}^{n} \rightarrow[0,1]$ be a strategy-proof SCF. Then, $f: \mathcal{S S P}^{n} \rightarrow[0,1]$ is group strategy-proof.

To see that in the statements of Propositions 1 and 2 tops-onlyness does not follow from strategy-proofness consider the $\mathrm{SCF} f: \mathcal{S P}^{n} \rightarrow[0,1]$ where for all $R \in \mathcal{S P}^{n}$,

$$
f(R)= \begin{cases}0 & \text { if } \#\left\{i \in N \mid 0 R_{i} 1\right\} \geq \#\left\{i \in N \mid 1 P_{i} 0\right\} \\ 1 & \text { otherwise. }\end{cases}
$$

Notice that $f$ is strategy-proof and anonymous but it is not tops-only. It also violates efficiency, unanimity, and ontoness. In the last section of the paper we will describe how our characterization includes this class of SCFs on the domain of symmetric singlepeaked preferences.

\subsection{Main intuition and definitions}

Consider Propositions 1 and 2 for the simplest case where $n=1 .{ }^{15}$ Figure 1 depicts the voting scheme $f:[0,1] \rightarrow[0,1]$ of a strategy-proof and tops-only SCF $f: \mathcal{S P} \rightarrow[0,1]$ with the two associated fixed ballots $0<p_{1}<p_{0}<1$. Observe that for any pair of fixed ballots $0 \leq p_{1} \leq p_{0} \leq 1$ the corresponding voting scheme $f:[0,1] \longrightarrow[0,1]$ is always increasing (i.e., $0 \leq x<y \leq 1$ implies $f(x) \leq f(y)$ ), continuous, and $r_{f}=\left[p_{1}, p_{0}\right]$. For any $n \geq 1$, a voting scheme $f:[0,1]^{n} \longrightarrow[0,1]$ is increasing if $f(t) \leq f\left(t^{\prime}\right)$ for all $t, t^{\prime} \in[0,1]^{n}$ such that $t_{i} \leq t_{i}^{\prime}$ for all $i \in N$.

\footnotetext{
${ }^{14}$ Barberà et al. (2010) give sufficient conditions defining domains of preferences under which strategyproofness is equivalent to group strategy-proofness. The domain of symmetric single-peaked preferences satisfies these sufficient conditions.

${ }^{15}$ When $n=1$ anonymity is vacuous. Indeed, we can uniquely identify the two fixed ballots of the propositions as $p_{1}=p_{\{1\}}$ and $p_{0}=p_{\emptyset}$.
} 


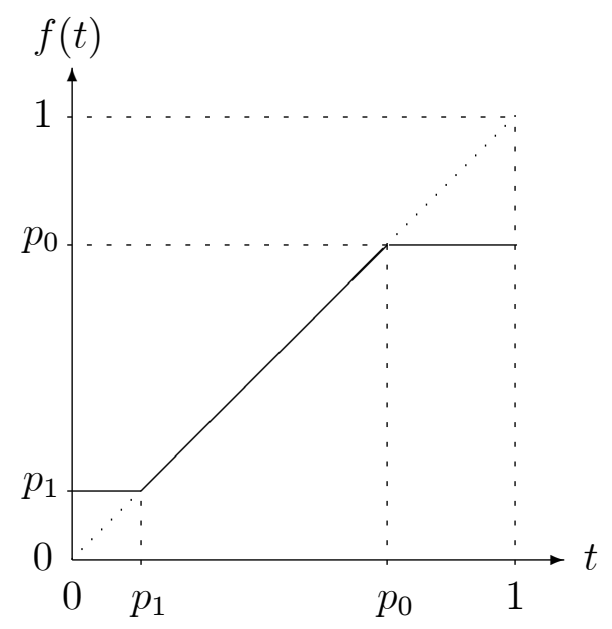

Figure 1

More generally, let $\mathcal{S}$ be a subset of $\mathcal{S P}$. A SCF $f: \mathcal{S}^{n} \rightarrow[0,1]$ is increasing if $f(R) \leq f\left(R^{\prime}\right)$ for all $R, R^{\prime} \in \mathcal{S}^{n}$ such that $t\left(R_{i}\right) \leq t\left(R_{i}^{\prime}\right)$ for all $i \in N$. By Proposition 2 the following remark holds.

Remark 2 Let $f: \mathcal{S P}^{n} \longrightarrow[0,1]$ be a strategy-proof and tops-only SCF. Then, its corresponding voting scheme $f:[0,1]^{n} \longrightarrow[0,1]$ is increasing and continuous.

Lemma 1 below states that, for any $n \geq 1$, any strategy-proof SCF is increasing on the domain of symmetric single-peaked preferences (observe that tops-only is not required explicitly since for each $x \in[0,1]$ there exists a unique $R_{i} \in \mathcal{S S P}$ such that $\left.t\left(R_{i}\right)=x\right)$.

Lemma 1 Let $f: \mathcal{S S P}^{n} \rightarrow[0,1]$ be a strategy-proof $S C F$. Then, $f$ is increasing.

Proof The statement follows from the iterated application of CLAIM A.

Claim A Let $f: \mathcal{S S P}^{n} \rightarrow[0,1]$ be a strategy-proof $S C F$. Let $t, t^{\prime} \in \mathcal{S S P}^{n}$ be such that for some $i \in N, t_{i}<t_{i}^{\prime}$ and $t_{-i}=t_{-i}^{\prime}$. Then, $f(t) \leq f\left(t^{\prime}\right)$.

Proof of Claim A Assume otherwise; that is, there exist $t, t^{\prime} \in \mathcal{S S P}^{n}$ and $i \in N$ such that

$$
t_{i}<t_{i}^{\prime}
$$

$t_{-i}=t_{-i}^{\prime}$ and $f\left(t^{\prime}\right)<f(t)$. We distinguish among six possible cases. The first three cases (i) $f\left(t^{\prime}\right)<f(t) \leq t_{i}<t_{i}^{\prime}$, (ii) $t_{i} \leq f\left(t^{\prime}\right)<f(t) \leq t_{i}^{\prime}$, and (iii) $f\left(t^{\prime}\right)<t_{i} \leq f(t) \leq t_{i}^{\prime}$ contradict strategy-proofness of $f$ since in all three cases $i$ manipulates $f$ at $t^{\prime}$ via $t_{i}$. The two cases (iv) $t_{i}<t_{i}^{\prime} \leq f\left(t^{\prime}\right)<f(t)$ and (v) $t_{i} \leq f\left(t^{\prime}\right) \leq t_{i}^{\prime} \leq f(t)$ contradict strategy-proofness of $f$ since in both cases $i$ manipulates $f$ at $t$ via $t_{i}^{\prime}$. The remaining 
case is (vi) $f\left(t^{\prime}\right) \leq t_{i}<t_{i}^{\prime} \leq f(t)$. Since $t_{i}, t_{i}^{\prime} \in \mathcal{S S P}$ and $f$ is strategy-proof,

$$
\begin{aligned}
f(t)-t_{i} & \leq t_{i}-f\left(t^{\prime}\right) \\
t_{i}^{\prime}-f\left(t^{\prime}\right) & \leq f(t)-t_{i}^{\prime} .
\end{aligned}
$$

Adding up,

$$
\begin{aligned}
f(t)-t_{i}+t_{i}^{\prime}-f\left(t^{\prime}\right) & \leq t_{i}-f\left(t^{\prime}\right)+f(t)-t_{i}^{\prime} \\
t_{i}^{\prime}-t_{i} & \leq t_{i}-t_{i}^{\prime} \\
t_{i}^{\prime} & \leq t_{i}
\end{aligned}
$$

a contradiction with $(2)$.

We have shown that the monotonicity of strategy-proof SCFs is preserved when we restrict the domain of single-peaked preferences to be symmetric. However, continuity (of its corresponding voting scheme) does not follow from strategy-proofness and topsonlyness in this smaller domain. Indeed, a special class of discontinuities may arise. It is very easy to understand why when $n=1$. First, take any $\tau, \delta \in(0,1)$ such that $\delta \leq \min \{\tau, 1-\tau\}$ and define the SCFs $f^{-}: \mathcal{S S P} \longrightarrow[0,1]$ and $f^{+}: \mathcal{S S P} \longrightarrow[0,1]$ where for each $t_{i} \in \mathcal{S S P}$,

$$
f^{-}\left(t_{i}\right)= \begin{cases}\tau-\delta & \text { if } t_{i} \leq \tau \\ \tau+\delta & \text { if } \tau<t_{i}\end{cases}
$$

and

$$
f^{+}\left(t_{i}\right)= \begin{cases}\tau-\delta & \text { if } t_{i}<\tau \\ \tau+\delta & \text { if } \tau \leq t_{i}\end{cases}
$$

In Figure 2 we depict $f^{-}$. Both $f^{-}$and $f^{+}$are strategy-proof on the domain of symmetric single-peaked preferences. At any $t_{i} \in \mathcal{S S P}$ such that either $t_{i}>\tau$ or $t_{i}<\tau$ agent $i$ can not manipulate them. Let $t_{i} \in \mathcal{S S P}$ be such that $t_{i}=\tau$. Then, $(\tau-\delta) I_{i}(\tau+\delta)$ since $(\tau-\delta)$ and $(\tau+\delta)$ are at the same distance $\delta$ to $\tau$. The function $f^{-}:[0,1] \longrightarrow[0,1]$ is left-continuous while the function $f^{+}:[0,1] \longrightarrow[0,1]$ is right-continuous. ${ }^{16}$ Observe that neither $f^{-}$nor $f^{+}$are strategy-proof on the domain of single-peaked preferences since, for instance, for $\tau=1 / 2, \delta=1 / 4$, and any $R_{i} \in \mathcal{S P}$ such that $t\left(R_{i}\right)=3 / 8$ and $3 / 4 P_{i} 1 / 4$ agent $i$ manipulates $f^{-}$and $f^{+}$at $R_{i}$ via any $R_{i}^{\prime}$ such that $t\left(R_{i}^{\prime}\right)=7 / 8$ since $f^{-}\left(R_{i}^{\prime}\right)=f^{+}\left(R_{i}^{\prime}\right)=3 / 4 P_{i} 1 / 4=f^{+}\left(R_{i}\right)=f^{-}\left(R_{i}\right)$.

\footnotetext{
${ }^{16} \mathrm{~A}$ function $g:[0,1] \longrightarrow[0,1]$ is left-continuous (respectively, right-continuous) if for all $x \in[0,1]$ and for any sequence $\left\{x_{m}\right\}_{m \in \mathbb{N}}$ such that $x_{m} \leq x$ (respectively, $x_{m} \geq x$ ) for all $m \in \mathbb{N},\left\{x_{m}\right\}_{m \in \mathbb{N}} \longrightarrow x$ implies $\left\{g\left(x_{m}\right)\right\}_{m \in \mathbb{N}} \longrightarrow g(x)$.
} 


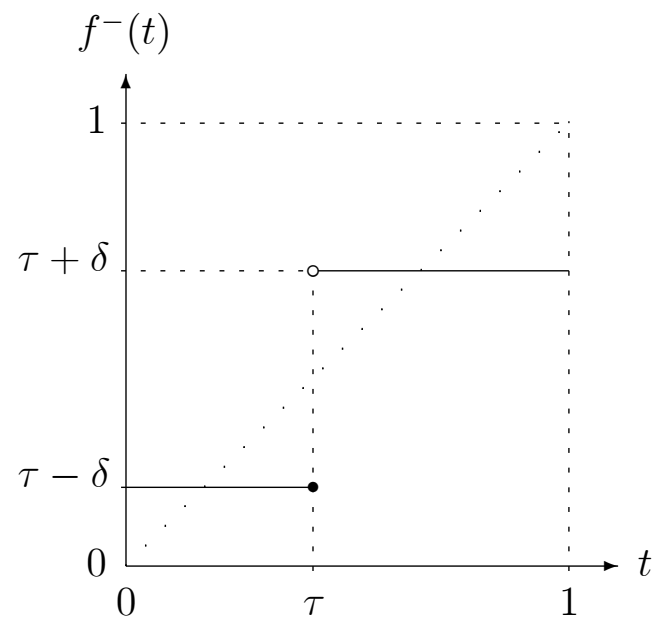

Figure 2

More generally, a strategy-proof $\mathrm{SCF} f: \mathcal{S S P} \longrightarrow[0,1]$ could have a countable number of discontinuities as long as the midpoint of each discontinuity jump is the discontinuity point itself; namely, for the point $d \in[0,1]$ where $f$ is discontinuous at $d$,

$$
d=\frac{\lim _{x \rightarrow d^{-}} f(x)+\lim _{x \rightarrow d^{+}} f(x)}{2}
$$

must hold, otherwise, $f$ is not strategy-proof. Thus, discontinuity jumps have to be symmetric around the discontinuity point.

As we will show in Section 4, the class of strategy-proof SCFs on the domain of symmetric single-peaked preferences is the class of generalized median voter schemes identified by Moulin (1980) plus the SCFs obtained after perturbing each generalized median voter scheme by admitting these very particular kind of discontinuities. We will call them disturbed minmax. Formally,

Definition 4 Let $\left\{p_{S}\right\}_{S \in 2^{N}}$ be a monotonic family of fixed ballots. A collection of open intervals $I=\left\{I_{m}\right\}_{m \in M}$, where $M$ is an indexation set, is a family of discontinuity jumps compatible with $\left\{p_{S}\right\}_{S \in 2^{N}}$ if:

(1) $M$ is countable,

(2) for all $m \in M, I_{m}=\left(a_{m}, b_{m}\right) \subset\left[p_{N}, p_{\emptyset}\right]$,

(3) for all $m, m^{\prime} \in M$ such that $m \neq m^{\prime}, I_{m} \cap I_{m^{\prime}}=\emptyset$,

(4) for all $S \in 2^{N}, p_{S} \notin \bigcup_{m \in M} I_{m}$.

Given a family of discontinuity jumps $I=\left\{I_{m}\right\}_{m \in M}$ we denote the midpoint of each open interval $I_{m}=\left(a_{m}, b_{m}\right)$ by $d_{m}=\frac{a_{m}+b_{m}}{2}$ and we preliminary perturb the identity function as follows. 
Definition 5 Given a family of discontinuity jumps $I=\left\{I_{m}\right\}_{m \in M}$, the corresponding perturbation function $\Pi^{I}:[0,1] \rightarrow[0,1]$ is defined as follows: for each $x \in[0,1]$,

$$
\Pi^{I}(x)= \begin{cases}x & \text { if } x \notin \bigcup_{m \in M} I_{m} \\ a_{m} & \text { if } x \in\left(a_{m}, d_{m}\right] \\ b_{m} & \text { if } x \in\left(d_{m}, b_{m}\right) .\end{cases}
$$

Let $I$ be a family of discontinuity jumps compatible with the monotonic family of fixed ballots $\left\{p_{S}\right\}_{S \in 2^{N}}$. A possible perturbation of the generalized median voter scheme associated to $\left\{p_{S}\right\}_{S \in 2^{N}}$ that preserves its strategy-proofness in the symmetric singlepeaked domain is as follows: for each $t=\left(t_{1}, \ldots, t_{n}\right) \in \mathcal{S S P}^{n}$,

$$
f\left(t_{1}, \ldots, t_{n}\right)=\Pi^{I}\left(\min _{S \in 2^{N}} \max _{i \in S}\left\{t_{i}, p_{S}\right\}\right) .
$$

We will show that these perturbed functions (of generalized median voter schemes) are the basis to characterize the class of all strategy-proof SCFs on the domain of symmetric single-peaked preferences.

Figure 3 illustrates the perturbation for the case $n=1, M=\{m\}$ and $I=\left\{I_{m}=\right.$ $\left.\left(a_{m}, b_{m}\right)\right\}$; i.e., $f(t)=\Pi^{I}\left(\operatorname{med}\left\{t, p_{1}, p_{0}\right\}\right)$.

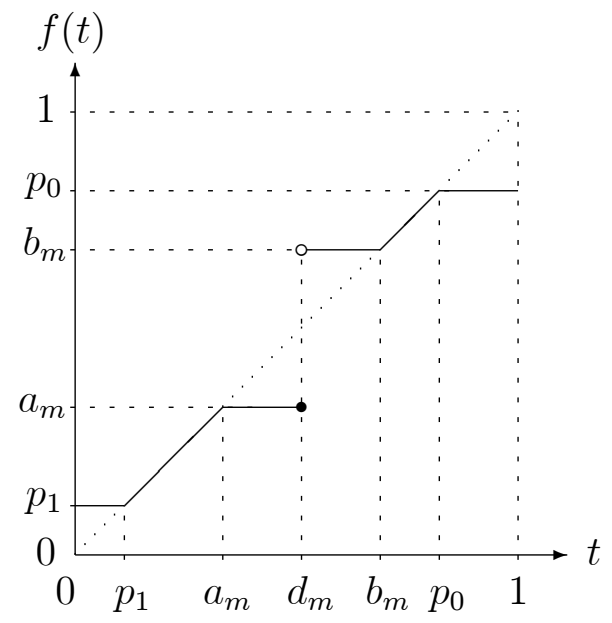

Figure 3

Notice that $\Pi^{I}$ arbitrarily assigns the value $a_{m}$ to the point $d_{m}$. If instead $\Pi^{I}\left(d_{m}\right)=$ $b_{m}$, the perturbed median voter scheme would still be strategy-proof. When $n=1$, there are just two ways of perturbing the generalized median voter scheme at each discontinuity jump while preserving its strategy-proofness. When $n>1$ the process of 
assigning values to the discontinuity points in a way that maintains strategy-proofness is more complex.

Figure 4 illustrates the perturbation of an anonymous SCF for the case $n=2$, $M=\{m\}, I=\left\{I_{m}\right\}$ and $0<p_{2}<a_{m}<d_{m}<b_{m}<p_{1}<p_{0}<1$; i.e., $f\left(t_{1}, t_{2}\right)=$ $\Pi^{I}\left(\operatorname{med}\left\{t_{1}, t_{2}, p_{2}, p_{1}, p_{0}\right\}\right)$. The tops of the two agents are measured on the axes and in bold-italic is represented the value of the SCF in each region. The bold line indicates the discontinuity points of the SCF.

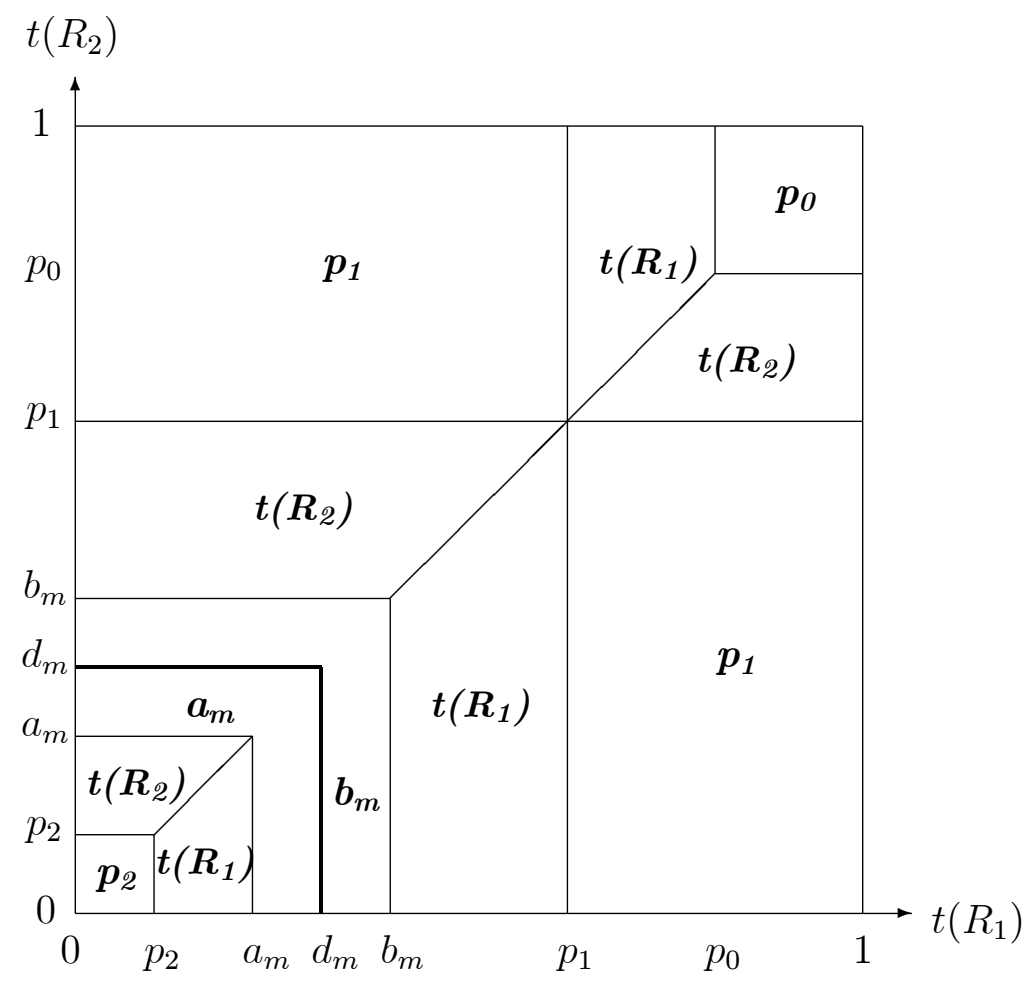

Figure 4

It is easy to see that if $\Pi^{I}$ had assigned the value $b_{m}$, instead of $a_{m}$, to $d_{m}$ the perturbation of the generalized median voter scheme would still have remained strategyproof on the domain of symmetric single-peaked preferences. But now there are more ways of assigning values to the discontinuity points that preserve the strategy-proofness of $f$. For the particular case depicted in Figure 4, the SCF would have remained strategyproof and anonymous if it had assigned the value $a_{m}$ to the points in the set $B_{1}=$ $\left\{\left(t_{1}, t_{2}\right) \in[0,1]^{2} \mid 0 \leq t_{1}<d_{m}\right.$ and $\left.t_{2}=d_{m}\right\}$, as well as to the points in the set $B_{2}=\left\{\left(t_{1}, t_{2}\right) \in[0,1]^{2} \mid t_{1}=d_{m}\right.$ and $\left.0 \leq t_{2}<d_{m}\right\}$, whereas it had assigned $b_{m}$ to the point $\left(d_{m}, d_{m}\right)$. Actually, if anonymity was not required then it could also have assigned the value $a_{m}$ to the points in $B_{1}$, and $b_{m}$ to the rest of points in $B_{2} \cup\left\{\left(d_{m}, d_{m}\right)\right\}$. However 
assigning the value $a_{m}$ to the point $\left(d_{m}, d_{m}\right)$ and $b_{m}$ to the rest of points in $B_{1} \cup B_{2}$ would violate strategy-proofness because at any profile $\left(t_{1}, d_{m}\right)$ with $0<t_{1}<d_{m}$ agent 1 could manipulate the SCF via $t_{1}^{\prime}=d_{m}$.

Intuitively, the perturbation of the generalized median voter scheme should preserve the increasing monotonicity of the SCF; otherwise, some agent could manipulate it at some profile. We next formalize all these possibilities.

Consider a generalized median voter scheme with its associated monotonic family of fixed ballots $\left\{p_{S}\right\}_{S \in 2^{N}}$. Let $I=\left\{I_{m}\right\}_{m \in M}$ be a family of discontinuity jumps compatible with $\left\{p_{S}\right\}_{S \in 2^{N}}$, and assume $M \neq \varnothing$. Fix $m \in M$ and define

$$
D_{m}=\left\{t=\left(t_{1}, \ldots, t_{n}\right) \in \mathcal{S} \mathcal{S} \mathcal{P}^{n} \mid \min _{S \in 2^{N}} \max _{i \in S}\left\{t_{i}, p_{S}\right\}=d_{m}\right\}
$$

namely, $D_{m}$ is the set of symmetric single-peaked preference profiles at which the generalized median voter scheme will select $d_{m}$ and thus the corresponding perturbation function $\Pi^{I}$ will generate a discontinuity point. We refer to any set $D_{m}$ as a discontinuity set. We want to determine the shape of the discontinuity sets because, in order to maintain strategy-proofness, we must preserve the increasing monotonicity of the function. To do that we need to track the agents with tops strictly below, equal, and strictly above $d_{m}$.

Note that, since no fixed ballot belongs to any discontinuity jump, if $t \in D_{m}$ then there is at least one agent $i \in N$ such that $t_{i}=d_{m}$.

For each $t \in D_{m}$ define the vector of extreme votes $e v^{m}(t)=\left(e v_{1}^{m}(t), \ldots, e v_{n}^{m}(t)\right) \in$ $\left\{0, d_{m}, 1\right\}^{n}$, where for each $i \in N$,

$$
e v_{i}^{m}(t)= \begin{cases}0 & \text { if } 0 \leq t_{i}<d_{m} \\ d_{m} & \text { if } t_{i}=d_{m} \\ 1 & \text { if } d_{m}<t_{i} \leq 1\end{cases}
$$

The vector $e v^{m}(t)$ describes at the profile $t$ the location of the top of each agent relative to $d_{m}$ (0 if it is strictly below, 1 if it is strictly above, and $d_{m}$ if it is exactly located at $\left.d_{m}\right)$. Let $E V\left(D_{m}\right)$ denote the set $\left\{e v^{m}(t) \mid t \in D_{m}\right\}$. Namely, the set $E V\left(D_{m}\right)$ describes all the extreme votes at which $d_{m}$ is chosen by the generalized median voter scheme associated to the monotonic family of fixed ballots $\left\{p_{S}\right\}_{S \in 2^{N}}$. Notice that since $\min _{S \in 2^{N}} \max _{i \in S}\left\{t_{i}, p_{S}\right\}=d_{m}$, if we reallocate the tops below $d_{m}$ to 0 , and the tops above $d_{m}$ to 1 , the minmax is not affected. Therefore, $\min _{S \in 2^{N}} \max _{i \in S}\left\{t_{i}, p_{S}\right\}=d_{m}=$ $\min _{S \in 2^{N}} \max _{i \in S}\left\{e v_{i}^{m}(t), p_{S}\right\}$.

We now turn to describe how strategy-proof SCFs on the symmetric single-peaked domain may choose between $a_{m}$ and $b_{m}$ at those profiles that induce a discontinuity at 
$d_{m}=\frac{a_{m}+b_{m}}{2}$. Define the preorder $\preceq$ on $\mathbb{R}^{n}$ as follows: for all $x, x^{\prime} \in \mathbb{R}^{n}$,

$$
x \preceq x^{\prime} \Leftrightarrow x_{i} \leq x_{i}^{\prime} \text { for all } i \in\{1, \ldots, N\}
$$

and, given $m \in M$, denote the restriction of $\preceq$ on the set $E V\left(D_{m}\right)$ by $\preceq_{m}$. Observe that the natural preorder $\preceq$ on $\mathbb{R}^{n}$ induces an incomplete, reflexive, and transitive binary relation $\preceq_{m}$ on $E V\left(D_{m}\right)$ with the property that $\widehat{e v}^{m} \preceq_{m} e v^{m}$ if and only if $e v^{m}$ represents a shift to the right of some of the extreme votes of $\widehat{e v}^{m}$. Thus, $\succeq_{m}$ can be read as the relation "to be more rightist than".

Let $Y_{m}$ be a non-empty subset of $E V\left(D_{m}\right)$. Denote by $X_{m}=U\left(Y_{m}\right)$ the upper contour set of $Y_{m}$ (according to $\preceq_{m}$ ) as

$$
X_{m}=U\left(Y_{m}\right)=\left\{e v^{m} \in E V\left(D_{m}\right) \mid \widehat{e v}^{m} \preceq_{m} e v^{m} \text { for some } \widehat{e v}^{m} \in Y_{m}\right\}
$$

By convention, set $U(\varnothing)=\varnothing$. Now, given $X_{m} \subseteq E V\left(D_{m}\right)$ with the property that $X_{m}=U\left(X_{m}\right)$, define $g^{X_{m}}: D_{m} \longrightarrow\left\{a_{m}, b_{m}\right\}$ as follows: for every $t \in D_{m}$,

$$
g^{X_{m}}(t)= \begin{cases}b_{m} & \text { if } e v^{m}(t) \in X_{m} \\ a_{m} & \text { otherwise. }\end{cases}
$$

The functions $g^{X_{m}}$ cover all different ways of assigning values $a_{m}$ and $b_{m}$ to the preference profiles that generate a discontinuity point at $d_{m}$ preserving the monotonicity of the perturbation. For each particular $m \in M$ there are many such functions because there are many subsets $X_{m} \subseteq E V\left(D_{m}\right)$ with the property that $X_{m}=U\left(X_{m}\right)$. Given a family of discontinuity jumps $I=\left\{I_{m}\right\}_{m \in M}$ we say that $\left\{X_{m}\right\}_{m \in M}$ is a family of tie-breaking sets of $M$ if for all $m \in M, X_{m} \subseteq E V\left(D_{m}\right)$ and $X_{m}=U\left(X_{m}\right)$.

\section{Characterization}

We are now ready to define disturbed minimax SCFs and state and prove that they constitute the class of all strategy-proof SCFs on the domain of symmetric single-peaked preferences.

\subsection{Definition and statement}

Definition 6 A SCF $f: \mathcal{S S P}^{n} \longrightarrow[0,1]$ is a disturbed minmax if there exist:

(1) a monotonic family of fixed ballots $\left\{p_{S}\right\}_{S \in 2^{N}}$;

(2) a family of discontinuity jumps $I=\left\{I_{m}\right\}_{m \in M}$ compatible with $\left\{p_{S}\right\}_{S \in 2^{N}}$; and 
(3) a family of tie-breaking sets $\left\{X_{m}\right\}_{m \in M}$ of $M$

such that, for all $t=\left(t_{1}, \ldots, t_{n}\right) \in \mathcal{S} \mathcal{S} \mathcal{P}^{n}$,

$$
f(t)= \begin{cases}\Pi^{I}\left(\min _{S \in 2^{N}} \max _{i \in S}\left\{t_{i}, p_{S}\right\}\right) & \text { if } \min _{S \in 2^{N}} \max _{i \in S}\left\{t_{i}, p_{S}\right\} \neq d_{m} \text { for all } m \in M \\ g^{X_{m}}\left(t_{1}, \ldots, t_{n}\right) & \text { if } \min _{S \in 2^{N}} \max _{i \in S}\left\{t_{i}, p_{S}\right\}=d_{m} \text { for some } m \in M\end{cases}
$$

Theorem 1 A SCF $f: \mathcal{S S P}^{n} \longrightarrow[0,1]$ is strategy-proof if and only if it is a disturbed minmax.

Before moving to the proof of Theorem 1 consider again the SCF $f$ defined in (1) but restricted to the domain of symmetric single-peaked preferences, where for all $R \in \mathcal{S S} \mathcal{P}^{n}$,

$$
f(R)= \begin{cases}0 & \text { if } \#\left\{i \in N \mid 0 R_{i} 1\right\} \geq \#\left\{i \in N \mid 1 P_{i} 0\right\} \\ 1 & \text { otherwise }\end{cases}
$$

Observe that for any $R_{i} \in \mathcal{S S P}, 0 R_{i} 1$ if and only if $t\left(R_{i}\right) \leq \frac{1}{2}$. It is easy to see that in the domain of single-peaked preferences $f$ is strategy-proof and anonymous but it is not tops-only. Hence, while it is excluded in Moulin (1980)'s characterization under the domain of single-peaked preferences stated above as Proposition 2, it has the following representation as a disturbed minmax under the domain of symmetric single-peaked preferences. Its family of monotonic fixed ballots is

$$
p_{S}= \begin{cases}0 & \text { if } \# S \geq\left\lceil\frac{n}{2}\right\rceil \\ 1 & \text { if } \# S<\left\lceil\frac{n}{2}\right\rceil\end{cases}
$$

where $\left\lceil\frac{n}{2}\right\rceil$ is the smallest integer larger or equal to $\frac{n}{2}$. The family $I$ of discontinuity jumps compatible with the monotonic family of fixed ballots contains only one discontinuity interval $I_{m}=\left(a_{m}, b_{m}\right)=(0,1)$ with $d_{m}=\frac{1}{2}$, and the tie-breaking set of $M=\{m\}$ is $X_{m}=\left\{e v \in\left\{0, \frac{1}{2}, 1\right\}^{n} \mid \#\left\{i \in N \mid e v_{i} \in\left\{0, \frac{1}{2}\right\}\right\}<\left\lceil\frac{n}{2}\right\rceil\right\}$.

\subsection{Preliminaries of the proof of Theorem 1}

We start with some additional notation. Given $x \in[0,1], S \subseteq N$ with $s=\# S$, and $t \in \mathcal{S} S \mathcal{P}^{n}$, define $x^{S} \equiv(\underbrace{x, \ldots, x}_{s-\text { times }})$ and $t_{S} \equiv\left(t_{j}\right)_{j \in S}$. Thus, $\left(x^{S}, t_{-S}\right) \equiv\left(y_{1}, \ldots, y_{n}\right)$, where $y_{j}=x$ if $j \in S$ and $y_{j}=t_{j}$ if $j \notin S$. Let $f: \mathcal{S S} \mathcal{P}^{n} \longrightarrow[0,1]$ be a SCF and $S \subseteq N$. Define the $\mathrm{SCF} \Delta_{f}^{S}:[0,1] \times \mathcal{S} \mathcal{S} \mathcal{P}^{n-s} \longrightarrow[0,1]$ as follows. For all $\left(x, t_{-S}\right) \in[0,1] \times \mathcal{S S P}^{n-s}$,

$$
\Delta_{f}^{S}\left(x, t_{-S}\right)=f\left(x^{S}, t_{-S}\right) .
$$


We will denote the diagonal function associated to $f$ by $\Delta_{f} \equiv \Delta_{f}^{N}$.

Given $t \in[0,1]^{n}$ and $x \in[0,1]$, define the subset of profiles of tops $\mathcal{C}_{t, x}$ as:

$$
\begin{array}{r}
\mathcal{C}_{t, x}=\left\{t^{\prime} \in \mathcal{S} \mathcal{S} \mathcal{P}^{n} \mid x \leq t_{i}^{\prime} \leq t_{i} \text { for all } i \text { such that } x \leq t_{i}\right. \text { and } \\
\left.t_{i} \leq t_{i}^{\prime} \leq x \text { for all } i \text { such that } t_{i} \leq x\right\}
\end{array}
$$

namely, $\mathcal{C}_{t, x}$ is the set of profiles $t^{\prime}$ with the property that the top $t_{i}^{\prime}$ of each agent $i$ lies between $t_{i}$ and $x$. Given a SCF $f: \mathcal{S S P}^{n} \longrightarrow[0,1]$, a subset $\mathcal{T} \subseteq \mathcal{S S P}^{n}$, and $x \in[0,1]$ the notation $\left.f\right|_{\mathcal{T}} \equiv x$ means that for all $t \in \mathcal{T}, f(t)=x$.

As a consequence of Remark 1 and Lemma 1 the following statements hold.

Remark 3 Let $f: \mathcal{S S P}^{n} \longrightarrow[0,1]$ be a strategy-proof SCF. Then,

(R3.1) $f$ is unanimous on its range $r_{f}$; namely, $x \in r_{f}$ implies $f\left(x^{N}\right)=x$;

(R3.2) for all $S \subseteq N, \Delta_{f}^{S}:[0,1] \times \mathcal{S S P}^{n-s} \longrightarrow[0,1]$ is strategy-proof; and

(R3.3) if $t \in \mathcal{S S P} \mathcal{P}^{n}$ is such that $f(t)=x$ then, $\left.f\right|_{\mathcal{C}_{t, x}} \equiv x$.

The two first statements follow from group strategy-proofness (Remark 1) and the last one from monotonicity (Lemma 1) and (R3.1).

We now state and prove the following three lemmata that will be useful in the proof of Theorem 1. Lemma 2 says that the range of a strategy-proof SCF and the range of its associated diagonal function coincide and it is a closed subset of $[0,1]$ (see also Zhou (1991)).

Lemma 2 Let $f: \mathcal{S S} \mathcal{P}^{n} \longrightarrow[0,1]$ be a strategy-proof $S C F$. Then, $r_{f}=r_{\Delta_{f}}$. Moreover, $r_{f}$ is closed.

Proof By definition of $\Delta_{f}, r_{\Delta_{f}} \subseteq r_{f}$. Take $x \in r_{f}$. Then, by (R3.1), $f\left(x^{N}\right)=x$. Thus, $x \in r_{\Delta_{f}}$. Let $\left\{x_{k}\right\} \rightarrow x$ be such that $x_{k} \in r_{f}$ for all $k \geq 1$ and assume $x \notin r_{f}$. Define $y=f\left(x^{N}\right) \neq x$ and let $x_{k}$ be such that $\left|x_{k}-x\right|<|y-x|$. By (R3.1), $f\left(x_{k}^{N}\right)=x_{k}$. Thus, $N$ manipulates $f$ at $x$ via $x_{k}$.

Lemmata 3 and $3^{\prime}$ roughly say that if a strategy-proof SCF is constant and equal to $x$ on one variable over some interval containing this constant $x$, but it is not constant over the whole interval $[0,1]$, then there is a discontinuity at some point $z$ and the discontinuity leaves indifferent the agent with top at $z$ (see Figures 2 and 3). In the proof of Theorem 1, $z$ will correspond to the midpoint $d_{m}$ of a discontinuity jump $I_{m}=\left(a_{m}, b_{m}\right)$, where $a_{m}=x$ and $b_{m}=2 z-x$.

Lemma 3 Let $f: \mathcal{S S P}^{n} \longrightarrow[0,1]$ be a strategy-proof $S C F$ with the property that there are $i \in N, x \in[a, b) \subset[0,1]$, and $t_{-i} \in \mathcal{S S P}^{n-1}$ such that

(3.1) $f\left(t_{i}, t_{-i}\right)=x$ for all $t_{i} \in[a, b)$ and 
(3.2) $f\left(1, t_{-i}\right)=y>x$.

Then, there exists $z \in\left[b, \frac{x+y}{2}\right]$ such that $f\left(\cdot, t_{-i}\right)$ is discontinuous at $z$ and

$$
\begin{aligned}
& \left.f\right|_{[a, z) \times\left\{t_{-i}\right\}} \equiv x \\
& \left.f\right|_{(z, 2 z-x] \times\left\{t_{-i}\right\}} \equiv 2 z-x .
\end{aligned}
$$

Proof Let $i \in N, x \in[a, b)$, and $t_{-i} \in \mathcal{S S P}^{n-1}$ be such that conditions (3.1) and (3.2) hold for $f$. First note that the interval $\left[b, \frac{x+y}{2}\right]$ is not empty since $b \leq \frac{x+y}{2}$ : If $b>\frac{x+y}{2}$ then $b$ would be closer to $y$ than to $x$ and for a small enough $\epsilon>0, i$ would manipulate $f$ at $\left(b-\epsilon, t_{-i}\right)$ via $t_{i}^{\prime}=1$.

Define $z=\sup \left\{t_{i} \in[0,1] \mid f\left(t_{i}, t_{-i}\right)=x\right\}$. Obviously $z \geq b>x$ and, by the monotonicity of $f, \lim _{t_{i} \rightarrow z^{-}} f\left(t_{i}, t_{-i}\right)=x$ and $\left.f\right|_{[a, z) \times\left\{t_{-i}\right\}} \equiv x$. We now prove that $\lim _{t_{i} \rightarrow z^{+}} f\left(t_{i}, t_{-i}\right)=2 z-x$. Suppose that $\lim _{t_{i} \rightarrow z^{+}} f\left(t_{i}, t_{-i}\right)<2 z-x$. Then, there exists $\epsilon>0$ such that $f\left(z+\epsilon, t_{-i}\right)<2 z-x-2 \epsilon$ and $f\left(z-\epsilon, t_{-i}\right)=x$. Either $f\left(z+\epsilon, t_{-i}\right) \geq z-\epsilon$, in which case $0 \leq f\left(z+\epsilon, t_{-i}\right)-(z-\epsilon)<(2 z-x-2 \epsilon)-(z-\epsilon)=(z-\epsilon)-f\left(z-\epsilon, t_{-i}\right)$, and hence, $i$ would manipulate $f$ at $\left(z-\epsilon, t_{-i}\right)$ via $t_{i}^{\prime}=z+\epsilon$. Or $f\left(z+\epsilon, t_{-i}\right)<z-\epsilon$, and therefore $f\left(z-\epsilon, t_{-i}\right)=x<f\left(z+\epsilon, t_{-i}\right)<z-\epsilon$, and $i$ would manipulate $f$ at $\left(z-\epsilon, t_{-i}\right)$ via $t_{i}^{\prime}=z+\epsilon$. Similarly, if $\lim _{t_{i} \rightarrow z^{+}} f\left(t_{i}, t_{-i}\right)>2 z-x$, there exists $\epsilon>0$ such that $f\left(z+\epsilon, t_{-i}\right)>2 z-x+2 \epsilon$ and $f\left(z-\epsilon, t_{-i}\right)=x$. But then $f\left(z+\epsilon, t_{-i}\right)-(z+\epsilon)>$ $(z+\epsilon)-x=(z+\epsilon)-f\left(z-\epsilon, t_{-i}\right)>0$ and hence, $i$ would manipulate $f$ at $\left(z+\epsilon, t_{-i}\right)$ via $z-\epsilon$. Thus, $\lim _{t_{i} \rightarrow z^{+}} f\left(t_{i}, t_{-i}\right)=2 z-x$ and $f\left(\cdot, t_{-i}\right)$ is discontinuous at $z$. Now by (R3.3), $\left.f\right|_{(z, 2 z-x] \times\left\{t_{-i}\right\}} \equiv 2 z-x$. Finally, by monotonicity of $f, 2 z-x \leq y$, and hence, $z \in\left[b, \frac{x+y}{2}\right]$.

Lemma $3^{\prime}$ Let $f: \mathcal{S S P}^{n} \longrightarrow[0,1]$ be a strategy-proof $S C F$ with the property that there are $i \in N, x \in(a, b] \subset[0,1]$, and $t_{-i} \in \mathcal{S S P}^{n-1}$ such that

$\left(3.1^{\prime}\right) f\left(t_{i}, t_{-i}\right)=x$ for all $t_{i} \in(a, b]$ and

$\left(3.2^{\prime}\right) f\left(0, t_{-i}\right)=y<x$.

Then, there exists $z \in\left[\frac{x+y}{2}, a\right]$ such that $f\left(\cdot, t_{-i}\right)$ is discontinuous at $z$ and

$$
\begin{aligned}
& \left.f\right|_{(z, b] \times\left\{t_{-i}\right\}} \equiv x \\
& \left.f\right|_{[2 z-x, z) \times\left\{t_{-i}\right\}} \equiv 2 z-x .
\end{aligned}
$$

Proof Omitted since it is symmetric to the proof of Lemma 3.

\subsection{Proof of Theorem 1}

It is easy to check that any disturbed minmax SCF is strategy-proof on the symmetric single-peaked domain. To see this notice that if $f$ is a disturbed minmax SCF, for all 
$t \in \mathcal{S} S \mathcal{P}^{n}$,

$$
\left|f(t)-\min _{S \in 2^{N}} \max _{j \in S}\left\{t_{j}, p_{S}\right\}\right|=\min \left\{\left|x-\min _{S \in 2^{N}} \max _{j \in S}\left\{t_{j}, p_{S}\right\}\right| \mid x \in r_{f}\right\} .
$$

Fix a profile $t \in \mathcal{S} \mathcal{S P} \mathcal{P}^{n}$ and an agent $i \in N$. If $t_{i}=\min _{S \in 2^{N}} \max _{j \in S}\left\{t_{j}, p_{S}\right\}$, then by (5) $i$ cannot benefit from reporting a different preference. Suppose that $t_{i}<\min _{S \in 2^{N}} \max _{j \in S}\left\{t_{j}, p_{S}\right\}$ (the case $t_{i}>\min _{S \in 2^{N}} \max _{j \in S}\left\{t_{j}, p_{S}\right\}$ is symmetric). The only way $i$ can affect the value of the SCF is by reporting a preference $t_{i}^{\prime}>\min _{S \in 2^{N}} \max _{j \in S}\left\{t_{j}, p_{S}\right\}$. Since disturbed minmax SCFs are increasing, $f\left(t_{i}^{\prime}, t_{-i}\right) \geq f(t)$. We distinguish between two cases:

Case 1: $f(t) \geq t_{i}$. Then $\left|f\left(t_{i}^{\prime}, t_{-i}\right)-t_{i}\right|=f\left(t_{i}^{\prime}, t_{-i}\right)-t_{i} \geq f(t)-t_{i}=\left|f(t)-t_{i}\right|$ and the deviation is not profitable.

Case 2: $f(t)<t_{i}<\min _{S \in 2^{N}} \max _{j \in S}\left\{t_{j}, p_{S}\right\}$. By the definition of the disturbed minmax, it must be that $f(t)=a_{m}$ for some $m \in M$ and $a_{m}<t_{i}<\min _{S \in 2^{N}} \max _{j \in S}\left\{t_{j}, p_{S}\right\} \leq d_{m}$. Hence, either $f\left(t_{i}^{\prime}, t_{-i}\right)=a_{m}=f(t)$, in which case the deviation is not profitable, or $f\left(t_{i}^{\prime}, t_{-i}\right) \geq b_{m}$ and $\left|f\left(t_{i}^{\prime}, t_{-i}\right)-t_{i}\right|=f\left(t_{i}^{\prime}, t_{-i}\right)-t_{i} \geq b_{m}-t_{i}>\frac{b_{m}-a_{m}}{2} \geq\left|f(t)-t_{i}\right|$, and again the deviation is not profitable.

Thus, any disturbed minmax SCF is strategy-proof.

Let $f: \mathcal{S S P}^{n} \longrightarrow[0,1]$ be a strategy-proof SCF. To show that $f$ is a disturbed min$\max$ we first have to identify its associated monotonic family of fixed ballots $\left\{p_{S}\right\}_{S \in 2^{N}}$, family $I=\left\{I_{m}\right\}_{m \in M}$ of discontinuity jumps compatible with $\left\{p_{S}\right\}_{S \in 2^{N}}$, and family of tiebreaking sets $\left\{X_{m}\right\}_{m \in M}$ of $M$. Then, we will show that $f$ coincides with the disturbed minmax SCF obtained by (4) in Definition 6, applied to all of them.

For each $S \in 2^{N}$, define its associated fixed ballot by setting

$$
p_{S} \equiv f\left(0^{S}, 1^{N \backslash S}\right)
$$

i.e., $p_{S}$ is the image of $f$ at the profile where all agents in $S$ have their top at 0 and all agents not in $S$ have their top at 1 .

Consider the diagonal function $\Delta_{f}: \mathcal{S S P} \longrightarrow[0,1]$ associated to $f$. By (R3.2) $\Delta_{f}$ is strategy-proof. Thus, by Lemma $1, \Delta_{f}$ is increasing and hence it has at most a countable number of discontinuities. ${ }^{17}$ Denote by $\left\{d_{m}\right\}_{m \in M}$ the discontinuity points of $\Delta_{f}$, where $M$ is a countable set. For each $m \in M$, define $a_{m}=\lim _{x \rightarrow d_{m}^{-}} \Delta_{f}(x)$, and $b_{m}=\lim _{x \rightarrow d_{m}^{+}} \Delta_{f}(x)$. Since $\Delta_{f}$ is discontinuous at $d_{m}$ and increasing on [0,1], $a_{m}$ and $b_{m}$ exist and $a_{m}<b_{m}$. By Lemma 2, $r_{\Delta_{f}}$ is closed and therefore $a_{m}, b_{m} \in r_{\Delta_{f}}$ and by

\footnotetext{
${ }^{17}$ Any real-valued monotone function of a real variable has at most a countable number of discontinuities. This result is due to Froda (1929) although in the literature it is widely used without Froda's name being mentioned.
} 
(R3.1), $\Delta_{f}\left(a_{m}\right)=a_{m}$ and $\Delta_{f}\left(b_{m}\right)=b_{m}$. Moreover, since $\Delta_{f}$ is strategy-proof, $d_{m}$ must be the midpoint of $I_{m} \equiv\left(a_{m}, b_{m}\right)$. Otherwise, if $d_{m}<\frac{a_{m}+b_{m}}{2}$, there would exist an $\epsilon>0$ such that $d_{m}<\frac{a_{m}+b_{m}}{2}-\epsilon$ and $\Delta_{f}\left(\frac{a_{m}+b_{m}}{2}-\epsilon\right) \geq b_{m}$, which would imply that $\Delta_{f}$ is manipulable at $\frac{a_{m}+b_{m}}{2}-\epsilon$ via $t^{\prime}=a_{m}$. Similarly, if $d_{m}>\frac{a_{m}+b_{m}}{2}$, there would exist an $\epsilon>0$ such that $d_{m}>\frac{a_{m}+b_{m}}{2}+\epsilon$ and $\Delta_{f}\left(\frac{a_{m}+b_{m}}{2}+\epsilon\right) \leq a_{m}$, which would imply that $\Delta_{f}$ is manipulable at $\frac{a_{m}+b_{m}}{2}+\epsilon$ via $t^{\prime}=b_{m}$.

Notice that the family of discontinuity jumps $I=\left\{I_{m}\right\}_{m \in M}$ is compatible with $\left\{p_{S}\right\}_{S \in 2^{N}}$ since:

(1) $M$ is countable.

(2) By the monotonicity of $\Delta_{f}, a_{m}=\Delta_{f}\left(a_{m}\right) \geq \Delta_{f}(0)=p_{N}$ and $b_{m}=\Delta_{f}\left(b_{m}\right) \leq$ $\Delta_{f}(1)=p_{\emptyset}$ and therefore $I_{m}=\left(a_{m}, b_{m}\right) \subset\left[p_{N}, p_{\emptyset}\right]$.

(3) By the monotonicity of $\Delta_{f}$ and the definition of $a_{m}$ and $b_{m}, I_{m} \cap I_{m^{\prime}}=\emptyset$ for any $m, m^{\prime} \in M, m^{\prime} \neq m$.

(4) Finally, by (6) and Lemma 2, for each $S \in 2^{N}, p_{S} \in r_{f}=r_{\Delta_{f}}, r_{f} \cap\left(a_{m}, b_{m}\right)=$ $r_{\Delta_{f}} \cap\left(a_{m}, b_{m}\right)=\emptyset$. Thus, for all $S \in 2^{N}, p_{S} \notin \bigcup_{m \in M} I_{m}$.

In fact,

$$
r_{f}=r_{\Delta_{f}}=\left[p_{N}, p_{\varnothing}\right] \backslash\left\{\bigcup_{m \in M} I_{m}\right\} .
$$

If $M$ is empty (i.e., $\Delta_{f}$ is continuos and its range is equal to $\left[p_{N}, p_{\varnothing}\right]$ ), the statement of Theorem 1 follows because $f$ is a generalized median voter scheme defined on the minimally rich domain $\mathcal{S S P}^{n}$ (see Theorem 1 in Berga and Serizawa (2000)). ${ }^{18}$

Assume $M$ is non-empty and fix $m \in M$. To identify the element $X_{m}$ in the family of tie-breaking sets of $M$, consider the previously defined discontinuity set

$$
D_{m}=\left\{t=\left(t_{1}, \ldots, t_{n}\right) \in \mathcal{S} \mathcal{S} \mathcal{P}^{n} \mid \min _{S \in 2^{N}} \max _{i \in S}\left\{t_{i}\right\}=d_{m}\right\},
$$

the set of profiles of extreme votes that induce $d_{m}$ through the minmax

$$
E V\left(D_{m}\right)=\left\{e v^{m}(t) \mid t \in D_{m}\right\},
$$

\footnotetext{
${ }^{18}$ Observe that all results in Berga and Serizawa (2000) refer only to onto SCFs. Hence, to be more precise with the application of their result, notice that the restriction of $\mathcal{S S P}$ on the interval $\left[p_{N}, p_{\varnothing}\right]$ is a symmetric single-peaked domain (on $\left[p_{N}, p_{\varnothing}\right]$ ) and it is a minimally rich domain (on $\left[p_{N}, p_{\varnothing}\right]$ ). Denote it by $\left.\mathcal{S S P}\right|_{\left[p_{N}, p_{\varnothing}\right]}$. Thus, we can identify the notation of Berga and Serizawa (2000) for the image set $Z=[\alpha, \beta]$ with our identified interval $\left[p_{N}, p_{\varnothing}\right]$ and apply their Theorem 1 to the SCF $f^{*}:\left(\left.\mathcal{S S P}\right|_{\left[p_{N}, p_{\varnothing}\right]}\right)^{n} \longrightarrow\left[p_{N}, p_{\varnothing}\right]$. Finally, observe that their generalized median voter schemes (defined through a left-coalition system) satisfy voter sovereignty and hence, $r_{f^{*}}=\left[p_{N}, p_{\varnothing}\right]$.
} 
and its associated preorder $\preceq_{m}$. Then, define

$$
X_{m}=\left\{e v^{m} \in E V\left(D_{m}\right) \mid f\left(e v^{m}\right)>d_{m}\right\} .
$$

By Lemma 1, $f$ is increasing and therefore $X_{m}$ coincides with its upper contour set relative to $\preceq_{m}$; i.e., $X_{m}=U\left(X_{m}\right)$.

So far we have identified from $f$ the monotonic family of fixed ballots $\left\{p_{S}\right\}_{S \in 2^{N}}$, the family $I=\left\{I_{m}\right\}_{m \in M}$ of discontinuity jumps compatible with $\left\{p_{S}\right\}_{S \in 2^{N}}$ (we are now assuming that $M \neq \varnothing$ ), and the family $\left\{X_{m}\right\}_{m \in M}$ of tie-breaking sets of $M$ (and hence, its corresponding family of tie-breaking functions $\left.\left\{g^{X_{m}}: D_{m} \longrightarrow\left\{a_{m}, b_{m}\right\}\right\}_{m \in M}\right)$. Given all of them, let $F$ be the SCF defined by condition (4) in Definition 6 . We want to show that $f=F$.

Let $t=\left(t_{1}, \ldots, t_{n}\right) \in \mathcal{S S P}^{n}$ be arbitrary. To show that $f(t)=F(t)$ define $q=$ $\min _{T \in 2^{N}} \max _{i \in T}\left\{t_{i}, p_{T}\right\}$. We distinguish among four different cases relating $q$, $t$ and $f(t)$.

CASE 1: $q \notin\left\{t_{1}, \ldots, t_{n}\right\}$.

Consider $S=\left\{i \in N \mid t_{i}<q\right\}$. Then $p_{S}=q$. To see that observe that if $p_{S}<q$ then $\max _{i \in S}\left\{t_{i}, p_{S}\right\}<q$ contradicting the definition of $q$. Further, since $q=\min _{T \in 2^{N}} \max _{i \in T}\left\{t_{i}, p_{T}\right\} \notin\left\{t_{1}, \ldots, t_{n}\right\}$, there exists $\bar{T} \in 2^{N}$, such that $p_{\bar{T}}=q$ and $t_{j}<p_{\bar{T}}$ for all $j \in \bar{T}$. But then, $\bar{T} \subseteq S$ and, by the monotonicity of $p=\left\{p_{T}\right\}_{T \in 2^{N}}$, $p_{S} \leq p_{\bar{T}}$. Therefore, by the definition of $q, p_{S}=p_{\bar{T}}=q$.

By the definition of $S$ and the assumption that $q \notin\left\{t_{1}, \ldots, t_{n}\right\}, t_{j}>p_{S}$ for all $j \notin S$. Then, $t \in \mathcal{C}_{\left(0^{S}, 1^{N \backslash S}\right), p_{S}}$ and, by (R3.3) and the definition of $p_{S},\left.f\right|_{\mathcal{C}_{\left(0^{S}, 1^{N \backslash S}\right), p_{S}}} \equiv p_{S}$. Therefore, $f(t)=p_{S}$.

Moreover, by (7), $p_{S} \notin \cup_{m \in M} I_{m}$. Hence, by (4) in Definition 6 and the definition of $\Pi^{I}$ in $(3), F(t)=\Pi^{I}\left(\min _{T \in 2^{N}} \max _{i \in T}\left\{t_{i}, p_{T}\right\}\right)=\min _{T \in 2^{N}} \max _{i \in T}\left\{t_{i}, p_{T}\right\}=p_{S}$. Thus, $f(t)=F(t)$.

CASE 2: $q=t_{i}$ for some $i \in N$ and $f(t)=t_{i}$.

If $t_{i}=f(t)$, then $t_{i} \in r_{f}$ and therefore, by (7), $t_{i} \notin \cup_{m \in M} I_{m}$. By (4) in Definition 6 and the definition of $\Pi^{I}$ in $(3), F(t)=\Pi^{I}\left(\min _{T \in 2^{N}} \max _{j \in T}\left\{t_{j}, p_{T}\right\}\right)=\Pi^{I}\left(t_{i}\right)=t_{i}$. Thus, $f(t)=F(t)$.

CASE 3: $q=t_{i}$ for some $i \in N, f(t) \equiv x \neq t_{i}$ and $t_{i} \notin \cup_{m \in M}\left\{d_{m}\right\}$.

To show that in this case $f(t)=F(t)$ we proceed in two steps. First we prove that $f(t)=f\left(t_{i}^{N}\right)$ and then we prove that $f\left(t_{i}^{N}\right)=F(t)$.

Step 1: $f(t)=f\left(t_{i}^{N}\right)$. 
Define $S_{i}^{<}=\left\{j \in N \mid t_{j}<t_{i}\right\}, S_{i}^{=}=\left\{j \in N \mid t_{j}=t_{i}\right\}$ and $S_{i}^{>}=\left\{j \in N \mid t_{j}>t_{i}\right\}$. We will denote $S_{i}^{\leq}=S_{i}^{<} \cup S_{i}^{=}$and $S_{i}^{\geq}=S_{i}^{>} \cup S_{i}^{=}$.

Because $t_{i}=\min _{T \in 2^{N}} \max _{j \in T}\left\{t_{j}, p_{T}\right\}$, it must be that $t_{i} \in\left[p_{S_{i}^{\leq}}, p_{S_{i}^{<}}\right]$. Otherwise, suppose first that $t_{i}<p_{S_{i}^{\leq}}$, and consider $T \in 2^{N}$. If $T \subset S_{i}^{\leq}$, we have that $t_{i}<p_{S_{i}^{\leq}} \leq p_{T}$, and therefore $t_{i}<\max _{j \in T}\left\{t_{j}, p_{T}\right\}$. If $T \cap S_{i}^{>} \neq \emptyset$, then by the definition of $S_{i}^{>}, \max _{j \in T}\left\{t_{j}, p_{T}\right\}>t_{i}$. Hence, we have a contradiction with $t_{i}=$ $\min _{T \in 2^{N}} \max _{j \in T}\left\{t_{j}, p_{T}\right\}$. Similarly, if $p_{S_{i}^{<}}<t_{i}$, then $\max _{j \in S_{i}^{<}}\left\{t_{j}, p_{S_{i}}\right\}<t_{i}$ again contradicting $t_{i}=\min _{T \in 2^{N}} \max _{j \in T}\left\{t_{j}, p_{T}\right\}$.

We now show that $f\left(t_{i}^{N}\right) \in\left[p_{S_{i}^{\leq}}, p_{S_{i}^{\leq}}\right]$. If $f\left(t_{i}^{N}\right)<p_{S_{i}^{\leq}} \leq t_{i}$, then $N$ manipulates $f$ at $t_{i}^{N}$ via $\left(0^{S_{i}^{\leq}}, 1^{S_{i}^{>}}\right)$since $f\left(0^{S_{i}^{\leq}}, 1^{S_{i}^{>}}\right)=p_{S_{i}^{\leq}}$, and if $t_{i} \leq p_{S_{i}^{<}}<f\left(t_{i}^{N}\right)$, then $N$ manipulates $f$ at $t_{i}^{N}$ via $\left(0^{S_{i}^{<}}, 1^{S_{i}^{\geq}}\right)$since $f\left(0^{S_{i}^{<}}, 1^{S_{i}^{\geq}}\right)=p_{S_{i}^{<}}$.

We prove that $f(t)=f\left(t_{i}^{N}\right)$ by contradiction. Suppose $f\left(t_{i}^{N}\right) \neq f(t)=x$. Then, either $x<f\left(t_{i}^{N}\right) \leq p_{S_{i}}$ or $p_{S_{i}^{\leq}} \leq f\left(t_{i}^{N}\right)<x$. The two cases are symmetric and therefore we omit the proof for the second case (which uses Lemma 3 ' instead of Lemma 3 ).

Suppose $x<f\left(t_{i}^{N}\right) \leq p_{S_{i}^{<}}$. The condition $x<f\left(t_{i}^{N}\right)$ implies $x<t_{i}$ since we are assuming that $x \neq t_{i}$ holds and if $x>t_{i}$, then $N$ would manipulate $f$ at $t_{i}^{N}$ via $t$. By (R3.3), the definition of $S_{i}^{\geq}$and $f(t)=x$,

$$
\Delta_{f}^{S_{i}^{\geq}}\left(\tau, t_{S_{i}^{<}}\right)=x \text { for all } \tau \in\left[x, t_{i}\right] .
$$

On the other hand, since $t_{j}<t_{i} \leq p_{S_{i}^{<}}$for all $j \in S_{i}^{<},\left(\tau^{S_{i}^{\geq}}, t_{S_{i}^{<}}\right) \in \mathcal{C}_{\left(0^{S_{i}^{<}}, S^{S_{i}}\right), p_{S_{i}^{<}}}$for all $\tau \in\left[p_{S_{i}^{<}}, 1\right]$, and therefore by (6) and (R3.3),

$$
\Delta_{f}^{S_{i}^{\geq}}\left(\tau, t_{S_{i}^{<}}\right)=p_{S_{i}^{<}} \text {for all } \tau \in\left[p_{S_{i}^{<}}, 1\right] .
$$

By Lemma 3, applied to the strategy-proof SCF $\Delta_{f}^{S_{i}^{\geq}}:[0,1] \times[0,1]^{S_{i}^{<}} \longrightarrow[0,1]$, where $[a, b)=\left[x, t_{i}\right)$ and $y=p_{S_{i}^{<}}$, there exists $z \in\left[t_{i}, \frac{x+p_{S_{i}^{<}}}{2}\right]$ such that $\Delta_{f}^{S_{i}^{\geq}}\left(\cdot, t_{S_{i}^{<}}\right)$is discontinuous at $z$ and

$$
\left.\Delta_{f}^{S_{i}^{\geq}}\right|_{[x, z) \times\left\{t_{S_{i}^{<}}\right\}} \equiv x \text { and }\left.\Delta_{f}^{S_{i}^{\geq}}\right|_{(z, 2 z-x] \times\left\{t_{S_{i}^{<}}\right\}} \equiv 2 z-x .
$$

Applying (R3.3) again, if $\tau \in(z, 2 z-x]$ and $t_{j}^{\prime} \in\left[t_{j}, 2 z-x\right]^{19}$ for all $j \in S_{i}^{<}$, then

$$
\Delta_{f}^{S_{i}^{\geq}}\left(\tau, t_{S_{i}^{<}}^{\prime}\right)=2 z-x \text {. }
$$

Note that $z$ is a discontinuity point of $\Delta_{f}$ as well. To see that observe that by (9), $f\left(w^{N}\right)=2 z-x$ for all $w \in(z, 2 z-x]$. On the other hand, $f(t)=x$, and hence, $x \in r_{f}$

\footnotetext{
${ }^{19}$ Notice that if $j \in S_{i}^{<}$then $t_{j}<t_{i} \leq 2 z-x$ and therefore the interval is well defined.
} 
and by (R3.1), $f\left(x^{N}\right)=x$. Assume that there exists $\hat{w} \in(x, z)$ such that $f\left(\hat{w}^{N}\right) \neq x$. By monotonicity of $f, x<f\left(\hat{w}^{N}\right) \leq 2 z-x$. Then, either $f\left(\hat{w}^{N}\right)=2 z-x$ and $N$ manipulates $f$ at $\hat{w}^{N}$ via $x^{N}$, or $f\left(\hat{w}^{N}\right)<2 z-x$ and for any $0<\epsilon<z-\hat{w}, N$ manipulates $f$ at $(z+\epsilon)^{N}$ via $\hat{w}^{N}$. Thus, $f\left(\hat{w}^{N}\right)=x$. Therefore, $\Delta_{f}$ has the property that

$$
\Delta_{f}(w)= \begin{cases}x & \text { if } w \in[x, z) \\ 2 z-x & \text { if } w \in(z, 2 z-x]\end{cases}
$$

This means that $\Delta_{f}$ is discontinuous at $z$ and hence there exists $m \in M$ such that $d_{m}=z$. Since under CASE $3, t_{i}$ is not a discontinuity point of $\Delta_{f}, t_{i} \neq z$ and therefore, by the definition of $z, t_{i}<z$.

By monotonicity of $f$ and $(9), f\left(t_{i}^{N}\right) \leq \Delta_{f}^{S_{i}^{\geq}}\left(z+\epsilon, t_{i}^{S_{i}^{<}}\right)=2 z-x$ for all sufficiently small $\epsilon>0$ (in the next paragraph we will find an upper bound for such $\epsilon$ 's). We want to show that the inequality is strict; i.e., $f\left(t_{i}^{N}\right)<2 z-x$ holds. Suppose $f\left(t_{i}^{N}\right)=2 z-x$; then, since $t_{i}<z$ can be re-written as $t_{i}-x<2 z-x-t_{i}$, this means that $N$ would manipulate $f$ at $t_{i}^{N}$ via $t$ which contradicts strategy-proofness of $f$.

To sum up, we have shown that if $x<f\left(t_{i}^{N}\right) \leq p_{S_{i}^{<}}$, then $f\left(t_{i}^{N}\right)<2 z-x$ and $\lim _{\tau \rightarrow z^{+}} \Delta_{f}^{S_{i}^{\geq}}\left(\tau, t_{i}^{S_{i}^{<}}\right)=2 z-x$. But then it is easy to see that for a small $\epsilon>0, S_{i}^{\geq}$ manipulates $f$ at $\left((z+\epsilon)^{S_{i}^{\geq}}, t_{i}^{S_{i}^{<}}\right)$via $t_{i}^{S_{i}^{\geq}}$. Namely, if $0<\epsilon<\frac{f\left(t_{i}^{N}\right)-x}{2}$, then $-(2 z-x-$ $(z+\epsilon))<f\left(t_{i}^{N}\right)-(z+\epsilon)<2 z-x-(z+\epsilon)$ where the first inequality is equivalent to the assumption $\epsilon<\frac{f\left(t_{i}^{N}\right)-x}{2}$, and the second inequality follows from $f\left(t_{i}^{N}\right)<2 z-x$. Therefore,

$$
\left|f\left(t_{i}^{N}\right)-(z+\epsilon)\right|<2 z-x-(z+\epsilon)
$$

which means that $S_{i}^{\geq}$manipulates $f$ at $\left((z+\epsilon)^{S_{i}^{\geq}}, t_{i}^{S_{i}^{<}}\right)$via $t_{i}^{S_{i}^{\geq}}$; a contradiction. This concludes the proof of Step 1 .

Step 2: $f\left(t_{i}^{N}\right)=F(t)$.

By strategy-proofness of $f, \Delta_{f}$ is strategy-proof and since $\Delta_{f}\left(t_{i}\right) \equiv f\left(t_{i}^{N}\right) \neq t_{i}$, by (R3.1), $t_{i} \notin r_{\Delta_{f}}$. By (7), there exists $m \in M$ such that $t_{i} \in\left(a_{m}, b_{m}\right)$. By (R3.1), $\Delta_{f}\left(a_{m}\right)=a_{m}$ and $\Delta_{f}\left(b_{m}\right)=b_{m}$. Since $\Delta_{f}$ is strategy-proof,

$$
x=\Delta_{f}\left(t_{i}\right)= \begin{cases}a_{m} & \text { if } a_{m}<t_{i}<d_{m} \\ b_{m} & \text { if } d_{m}<t_{i}<b_{m},\end{cases}
$$

which coincides with the value of $F(t)=\Pi^{I}\left(\min _{T \in 2^{N}} \max _{j \in T}\left\{t_{j}, p_{T}\right\}=\Pi^{I}\left(t_{i}\right)=x\right.$. Thus, $f\left(t_{i}^{N}\right) \equiv \Delta_{f}\left(t_{i}\right)=F(t)$. This concludes the proof of Step 2.

Putting together Step 1 and Step 2, we have shown that $f(t)=F(t)$. 
CASE 4: $q=t_{i}$ for some $i \in N, f(t) \equiv x \neq t_{i}$ and $t_{i}=d_{m}$ for some $m \in M$.

Denote by $I_{m}=\left(a_{m}, b_{m}\right)$ the discontinuity jump corresponding to $d_{m}$. Denote $S_{m}^{=}=$ $\left\{j \in N \mid t_{j}=d_{m}\right\}, S_{m}^{<}=\left\{j \in N \mid t_{j}<d_{m}\right\}$ and $S_{m}^{>}=\left\{j \in N \mid t_{j}>d_{m}\right\}$, and let $\epsilon$ be such that $0<\epsilon<\min _{j \in S_{m}^{<}, k \in S_{m}^{>}}\left\{d_{m}-a_{m}, d_{m}-t_{j}, t_{k}-d_{m}\right\}$. Given this $\epsilon>0$, consider the two profiles of tops $t^{\epsilon-}=\left(t_{S_{m}^{<}},\left(d_{m}-\epsilon\right)^{S_{m}^{=}}, t_{S_{m}^{>}}\right)$and $t^{\epsilon+}=\left(t_{S_{m}^{<}},\left(d_{m}+\epsilon\right)^{S_{m}^{=}}, t_{S_{m}^{>}}\right)$. By construction of $t^{\epsilon-}$ and $t^{\epsilon+}$, the fact that $t_{i}=\min _{T \in 2^{N}} \max _{j \in T}\left\{t_{j}, p_{T}\right\}$, and since $p_{T} \notin I_{m}$ for all $T \in 2^{N}, \min _{T \in 2^{N}} \max _{j \in T}\left\{t_{j}^{\epsilon-}, p_{T}\right\}=d_{m}-\epsilon$ and $\min _{T \in 2^{N}} \max _{j \in T}\left\{t_{j}^{\epsilon+}, p_{T}\right\}=d_{m}+\epsilon$. Both $d_{m}-\epsilon$ and $d_{m}+\epsilon$ belong to $I_{m}$ and therefore they do not belong to $r_{f}$. Moreover, since $I_{m} \cap I_{m^{\prime}}=\emptyset$, neither $d_{m}-\epsilon$ nor $d_{m}+\epsilon$ are discontinuity points of $\Delta_{f}$. We are therefore under the assumptions of CASE 3, and by Step1:

$$
\begin{aligned}
& f\left(t^{\epsilon-}\right)=\Delta_{f}\left(d_{m}-\epsilon\right)=a_{m} \\
& f\left(t^{\epsilon+}\right)=\Delta_{f}\left(d_{m}+\epsilon\right)=b_{m},
\end{aligned}
$$

where the second equality in both statements follow from the strategy-proofness of $\Delta_{f}$. By monotonicity, $f\left(t^{\epsilon-}\right) \leq f(t) \leq f\left(t^{\epsilon+}\right)$, which together with $(7)$ implies that $f(t) \in$ $\left\{a_{m}, b_{m}\right\}$. Thus, we have shown that if $t$ is such that $\min _{T \in 2^{N}} \max _{j \in T}\left\{t_{j}, p_{T}\right\}=t_{i}=d_{m}$ for some $m \in M$ then,

$$
f(t) \in\left\{a_{m}, b_{m}\right\} .
$$

To show that $f(t)=F(t)$, assume first that $t$ is such that $e v^{m}(t) \notin X_{m}$. By definition of $F, F(t)=a_{m}$. Since $e v^{m}(t) \notin X_{m}$, by (8), $f\left(0^{S_{m}^{<}}, d_{m}^{S_{m}^{=}}, 1^{S_{m}^{>}}\right) \leq d_{m}$ which means, by (7), that $f\left(0^{S_{m}^{<}}, d_{m}^{S_{m}^{=}}, 1^{S_{m}^{>}}\right) \leq a_{m}$. Moreover, $t^{\prime}=\left(0^{S_{m}^{<}}, d_{m}^{S_{m}^{=}}, 1^{S_{m}}\right)$ is such that $\min _{T \in 2^{N}} \max _{j \in T}\left\{t_{j}^{\prime}, p_{T}\right\}=d_{m}$ and, by (11), $f\left(0^{S_{m}^{<}}, d_{m}^{S_{m}^{\bar{m}}}, 1^{S_{m}^{>}}\right)=a_{m}$. By (R3.3),

$$
f\left(0^{S_{m}^{<}}, d_{m}^{S_{m}^{=}}, t_{S_{m}^{>}}\right)=a_{m}
$$

If $S_{m}^{<}=\varnothing$, then $\left(0^{S_{m}^{<}}, d_{m}^{S_{m}^{\bar{m}}}, t_{S_{m}^{>}}\right)=t$, and $f(t)=a_{m}$. If $S_{m}^{<} \neq \varnothing$ then $f(t)=a_{m}$ or otherwise $S_{m}^{<}$manipulates $f$ at $t$ via $0^{S_{m}^{<}}$. Thus, we have shown that $f(t)=a_{m}=F(t)$. Symmetrically, we can show that if $t$ is such that $e v^{m}(t) \in X_{m}$ then $f(t)=F(t)=b_{m}$.

This finishes the proof of Theorem 1 .

\section{Final remarks}

As direct consequences of Theorem 1, Corollaries 1, 2 and 3 below characterize three relevant subclasses of strategy-proof SCFs on the domain of symmetric single-peaked preferences. 


\subsection{Anonymity and efficiency}

Corollaries 1 and 2 characterize two nested subclasses: the class of strategy-proof and anonymous SCFs (Corollary 1) and the class of strategy-proof, anonymous and efficient SCFs (Corollary 2).

To state Corollary 1 we first need to translate the definitions of extreme votes and tie-breaking sets of $M$ to the anonymous case. Consider the family of $n+1$ fixed ballots

$0 \leq p_{n} \leq \ldots \leq p_{1} \leq p_{0} \leq 1$ associated to a median voter scheme and let $m \in M$. The set of profiles at which the median voter scheme will select $d_{m}$ is

$$
\widetilde{D}_{m}=\left\{t=\left(t_{1}, \ldots, t_{n}\right) \in \mathcal{S S P}^{n} \mid \operatorname{med}\left\{t_{1}, \ldots, t_{n}, p_{n}, \ldots, p_{0}\right\}=d_{m}\right\} .
$$

By anonymity, we only need to track the number of agents with tops strictly below, equal, and strictly above $d_{m}$. Hence, for each $t=\left(t_{1}, \ldots, t_{n}\right) \in \mathcal{S} \mathcal{S} \mathcal{P}^{n}$, define the triple $l^{m}(t)=\left(l_{<}^{m}(t), l_{=}^{m}(t), l_{>}^{m}(t)\right)$ where:

(1) $l_{<}^{m}(t)=\#\left\{i \in N \mid t_{i}<d_{m}\right\}$,

(2) $l_{=}^{m}(t)=\#\left\{i \in N \mid t_{i}=d_{m}\right\}$, and

(3) $l_{>}^{m}(t)=\#\left\{i \in N \mid t_{i}>d_{m}\right\}$.

Observe that $l_{<}^{m}(t)+l_{=}^{m}(t)+l_{>}^{m}(t)=n$ and since fixed ballots do not belong to any discontinuity jump, if $t \in \widetilde{D}_{m}$ then, there is $i \in N$ such that $t_{i}=d_{m}$ (i.e., $l_{=}^{m}(t) \geq 1$ ). Let $\nabla^{n}=\left\{(x, y, z) \in\{0,1, \ldots, n\}^{3} \mid x+y+z=n\right.$ and $\left.y \geq 1\right\}$ be the set of triples with positive integer components adding up to $n$ and whose middle component is equal or larger than 1 and define $L\left(\widetilde{D}_{m}\right)=\left\{l^{m}(t) \in \nabla^{n} \mid t=\left(t_{1}, \ldots, t_{n}\right) \in \widetilde{D}_{m}\right\}$; namely, $L\left(\widetilde{D}_{m}\right)$ describes all anonymous distributions of tops (number of tops strictly below $d_{m}$, number of tops at $d_{m}$, number of tops strictly above $d_{m}$ ) at which the median voter selects $d_{m}$. Define the preorder $\preceq$ on $\{0,1, \ldots, n\}^{3}$ as follows: for all $(x, y, z),\left(x^{\prime}, y^{\prime}, z^{\prime}\right) \in\{0,1, \ldots, n\}^{3}$,

$$
\left(x^{\prime}, y^{\prime}, z^{\prime}\right) \preceq(x, y, z) \Leftrightarrow z^{\prime} \leq z \text { and } x^{\prime} \geq x .
$$

Denote the restriction of the preorder $\widetilde{\preceq}$ on the set $L\left(\widetilde{D}_{m}\right)$ by $\widetilde{ }_{m}$ and let $\widetilde{Y}_{m}$ be a nonempty subset of $L\left(\widetilde{D}_{m}\right)$. Denote by $\widetilde{X}_{m}=U\left(\widetilde{Y}_{m}\right)$ the upper contour set of $\widetilde{Y}_{m}$ (according to $\left.\widetilde{\preceq}_{m}\right)$ as the set of triples in $L\left(\widetilde{D}_{m}\right)$ such that they are more rightist than some triple in $\widetilde{Y}_{m}$; namely,

$$
\widetilde{X}_{m}=U\left(\widetilde{Y}_{m}\right)=\left\{\left(l_{<}, l_{=}, l_{>}\right) \in L\left(\widetilde{D}_{m}\right) \mid(x, y, z) \widetilde{\preceq}_{m}\left(l_{<}, l_{=}, l_{>}\right) \text {for some }(x, y, z) \in \widetilde{Y}_{m}\right\} .
$$

By convention, set $U(\varnothing)=\varnothing$. Given $\widetilde{X}_{m} \subseteq L\left(\widetilde{D}_{m}\right)$ with the property that $\widetilde{X}_{m}=$ $U\left(\widetilde{X}_{m}\right)$, define $g^{\widetilde{X}_{m}}: \widetilde{D}_{m} \longrightarrow\left\{a_{m}, b_{m}\right\}$ as follows: for every $t \in \widetilde{D}_{m}$,

$$
g^{\widetilde{X}_{m}}(t)= \begin{cases}b_{m} & \text { if } l^{m}(t) \in \widetilde{X}_{m} \\ a_{m} & \text { otherwise }\end{cases}
$$


Given a family of discontinuity jumps $I=\left\{I_{m}\right\}_{m \in M}$ we say that $\left\{\widetilde{X}_{m}\right\}_{m \in M}$ is an anonymous family of tie-breaking sets of $M$ if for all $m \in M, \widetilde{X}_{m} \subseteq L\left(\widetilde{D}_{m}\right)$ and $\widetilde{X}_{m}=U\left(\widetilde{X}_{m}\right)$. Definition 7 A SCF $f: \mathcal{S S P}^{n} \longrightarrow[0,1]$ is a disturbed median if there exist:

(1) a family of $n+1$ fixed ballots $0 \leq p_{n} \leq \ldots \leq p_{1} \leq p_{0} \leq 1$;

(2) a family of discontinuity jumps $I=\left\{I_{m}\right\}_{m \in M}$ compatible with $p_{n}, \ldots, p_{1}, p_{0}$; and

(3) an anonymous family of tie-breaking sets $\left\{\widetilde{X}_{m}\right\}_{m \in M}$ of $M$ such that, for all $t=\left(t_{1}, \ldots, t_{n}\right) \in \mathcal{S} \mathcal{S} \mathcal{P}^{n}$,

$f(t)= \begin{cases}\Pi^{I}\left(\operatorname{med}\left\{t_{1}, \ldots, t_{n}, p_{n}, \ldots, p_{0}\right\}\right) & \text { if } \operatorname{med}\left\{t_{1}, \ldots, t_{n}, p_{n}, \ldots, p_{0}\right\} \neq d_{m} \text { for all } m \in M \\ g^{\widetilde{X}_{m}}\left(t_{1}, \ldots, t_{n}\right) & \text { if } \operatorname{med}\left\{t_{1}, \ldots, t_{n}, p_{n}, \ldots, p_{0}\right\}=d_{m} \text { for an } m \in M\end{cases}$

Corollary 1 A SCF $f: \mathcal{S S P}^{n} \longrightarrow[0,1]$ is strategy-proof and anonymous if and only if it is a disturbed median.

Corollary $2 A S C F f: \mathcal{S S P}^{n} \longrightarrow[0,1]$ is strategy-proof, anonymous, and efficient if and only if it is a median voter scheme with the property that $p_{n}=0$ and $p_{0}=1$.

Efficiency requires that $f$ respects unanimity and hence, $r_{f}=[0,1]$. Thus, (i) its associated family of $n+1$ fixed ballots has the property that $0=p_{n} \leq p_{n-1} \leq \ldots \leq p_{0}=1$ and (ii) the family of discontinuity sets $M$ is empty. Observe that since $p_{n}=0$ and $p_{0}=1$ they cancel each other out in the computation of the median at any profile $t$ and therefore, the generalized median voter scheme can also be described as the median of the $n$ tops and the $n-1$ fixed ballots $p_{n-1} \leq \ldots \leq p_{1}$. This corresponds to Moulin (1980)'s characterization of the class of strategy-proof, anonymous and efficient SCFs on the domain of single-peaked preferences. Thus, the reduction of the domain does not generate in this case new strategy-proof, anonymous and efficient SCFs.

\subsection{Feasibility constraints}

Our result has important implications for the design of strategy-proof SCFs on the domain of symmetric single-peaked preferences under feasibility constraints. Often, some subsets of alternatives (although conceivable) can not be chosen due to feasibility constraints. Then, discontinuities are compulsory rather than pathological because discontinuity jumps on the range of strategy-proof SCFs are necessary. Our result precisely describes their nature and how the strategy-proof SCF may select its value at these discontinuity points. However, if $f$ is a strategy-proof and discontinuous SCF then, $r_{f} \subsetneq[0,1]$ and hence, $f$ will not be efficient; in particular, $f$ will not respect unanimity. 
SCFs that are not efficient but they are efficient relative to the feasible set of alternatives are specially interesting. Thus, let $A \subsetneq[0,1]$ be a closed set of feasible alternatives. ${ }^{20}$ A SCF $f: \mathcal{S S P}^{n} \longrightarrow[0,1]$ is efficient relative to $A$ if $r_{f} \subseteq A$ and for all $R \in \mathcal{S S P}^{n}$ there is no $z \in A$ such that, for all $i \in N, z R_{i} f(R)$ and $z P_{j} f(R)$ for some $j \in N$. The following result follows from Theorem 1.

Corollary 3 Let $A$ be a closed subset of $[0,1]$. A SCF $f: \mathcal{S S P}^{n} \longrightarrow[0,1]$ is strategyproof and efficient relative to $A$ if and only if it is a disturbed minmax with $r_{f}=A$.

Note that the requirement $r_{f}=A$ imposes certain conditions on the monotonic family of fixed ballots $\left\{p_{S}\right\}_{S \in 2^{N}}$ and on the discontinuity jumps. For instance $p_{N}=\min \{x \in A\}$, $p_{\varnothing}=\max \{x \in A\}$ and $p_{S} \in A$ for all $S \in 2^{N}$. Moreover since $A$ is closed the set $\left[p_{N}, p_{\varnothing}\right] \backslash A$ is open and therefore it can be written as a countable and disjoint union of open intervals: $\left[p_{N}, p_{\varnothing}\right] \backslash A=\cup_{m \in M} I_{m}$ where $I_{m}$ is an open interval for all $m \in M$ and $I_{m} \cap I_{m^{\prime}}=\varnothing$ for all $m, m^{\prime} \in M$. This representation is unique up to permutations in $M$, and in fact the requirement $r_{f}=A$ implies that the family of discontinuity jumps compatible with $\left\{p_{S}\right\}_{S \in 2^{N}}$ is exactly $I=\left\{I_{m}\right\}_{m \in M}$.

As an illustration of Corollary 3 , suppose that the set of feasible alternatives is $A=$ $\{0\} \cup\{0.1\} \cup[0.2,0.8] \cup\{0.9\}$. In that case the only general requirements on the fixed ballots are that $p_{N}=0, p_{\varnothing}=0.9$ and $p_{S}$ has to belong to $A$ for all $S \in 2^{N}$. The family of discontinuity jumps is given by $I_{1}=(0,0.1), I_{2}=(0.1,0.2)$, and $I_{3}=(0.8,0.9)$, and therefore the discontinuity points are $d_{1}=0.05, d_{2}=0.15$ and $d_{3}=0.85$. To proceed with the illustration and in order to design a particular strategy-proof and anonymous SCF $f$ whose range $r_{f}$ be equal to $A$ let $N=\{1,2,3\}$ be the set of agents and let $p_{3}=p_{2}=0$ and $p_{1}=p_{0}=0.9$ be the family of four fixed ballots. In this particular case the ballots cancel each other and hence, for all $\left(t_{1}, t_{2}, t_{3}\right) \in \mathcal{S S P}^{3}, \operatorname{med}\left\{t_{1}, t_{2}, t_{3}, 0,0,0.9,0.9\right\}=$ $\operatorname{med}\left\{t_{1}, t_{2}, t_{3}\right\}$. For each discontinuity point $d_{m}$ the set $L\left(\widetilde{D}_{m}\right)$ consists of four triplets: $L\left(\widetilde{D}_{m}\right)=\{(1,2,0),(0,3,0),(1,1,1),(0,2,1)\}$ where for example, the triplet $(1,2,0)$ means that one top is strictly below $d_{m}$ and the remaining two tops are exactly equal to $d_{m}$. Note, that in all the four cases the median of the tops coincides with $d_{m}$, and hence all the profiles of tops that are represented by $L\left(\widetilde{D}_{m}\right)$ result in discontinuity points. Moreover, and since $L\left(\widetilde{D}_{1}\right)=L\left(\widetilde{D}_{2}\right)=L\left(\widetilde{D}_{3}\right), \widetilde{\preceq}_{1}=\widetilde{\preceq}_{2}=\widetilde{\preceq}_{3}$ as well. Denote it by $\check{\preceq}^{\prime}$ and ob-

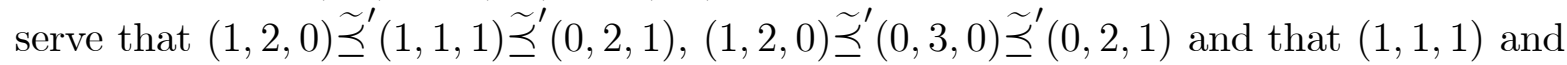
$(0,3,0)$ are not comparable by $\breve{\Upsilon}^{\prime}$. To assign a value to the SCF on these discontinuity points preserving the monotonicity of the SCF $f$ we need to select for each $d_{m}$ a tie-

\footnotetext{
${ }^{20}$ Remember that, by Lemma 2, strategy-proof SCFs have a closed range.
} 
breaking set $\widetilde{X}_{m}$ such that $\widetilde{X}_{m}=U\left(\widetilde{X}_{m}\right)$. Given $L\left(\widetilde{D}_{m}\right)$, there are six different ways of doing so: $\widetilde{X}_{m} \in\{\emptyset,\{(0,2,1)\},\{(1,1,1),(0,2,1)\},\{(0,3,0),(0,2,1)\},\{(1,1,1),,(0,3,0),(0,2,1)\}$, $\left.L\left(\widetilde{D}_{m}\right)\right\}$. For instance, choose $\widetilde{X}_{1}=\{(1,1,1),(0,2,1)\}, \widetilde{X}_{2}=\{(0,2,1)\}$, and $\widetilde{X}_{3}=$ $L\left(\widetilde{D}_{m}\right)$. Thus, the disturbed median $f$ that we may define applying Definition 7 to the family of four fixed ballots $0=p_{3}=p_{2}<p_{1}=p_{0}=0.9$, the family of discontinuity jumps $I_{1}=(0,0.1), I_{2}=(0.1,0.2)$, and $I_{3}=(0.8,0.9)$, and the anonymous family of tie-breaking sets $\widetilde{X}_{1}=\{(1,1,1),(0,2,1)\}, \widetilde{X}_{2}=\{(0,2,1)\}$, and $\widetilde{X}_{3}=L\left(\widetilde{D}_{3}\right)$ has range equal to $A$ and it is efficient relative to $A$. The disturbed median $f$ could also be defined as follows. For all $t=\left(t_{1}, t_{2}, t_{3}\right) \in \mathcal{S} \mathcal{S P}^{3}$, and after setting $y \equiv \operatorname{med}\left\{t_{1}, t_{2}, t_{3}\right\}$,

$$
f(t)= \begin{cases}0 & \text { if } y<0.05 \text { or } y=0.05 \text { and } \#\left\{i \mid t_{i} \leq 0.05\right\}=3 \\ 0.1 & \text { if } y=0.05 \text { and } \#\left\{i \mid t_{i} \leq 0.05\right\}<3 \text { or } 0.05<y<0.15 \\ & \text { or } y=0.15 \text { and either } \exists j \text { s.t. } t_{j}<0.15 \text { or } t_{1}=t_{2}=t_{3}=0.15 \\ 0.2 & \text { if } y=0.15 \text { and } \#\left\{i \mid t_{i} \geq 0.15\right\}=3 \text { and } \exists j \text { s.t. } t_{j}>0.15 \\ & \text { or } 0.15<y<0.2 \\ y & \text { if } 0.2 \leq y \leq 0.8 \\ 0.8 & \text { if } 0.8<y<0.85 \\ 0.9 & \text { if } y \geq 0.85 .\end{cases}
$$

The complexity of this description indicates the usefulness of Theorem 1's characterization.

Finally, by Remark 1, the four statements above (Theorem 1 and Corollaries 1, 2 and 3) also hold after replacing strategy-proofness by group strategy-proofness.

\section{Acknowledgments}

We thank Gilat Levy, William Thomson, two referees, the participants of the Bag Lunch Workshop on Game Theory and Social Choice at the UAB, and the participants of the Theory Work in Progress Seminar at LSE for their helpful critical comments and suggestions. Support for the research of Jordi Massó was received through the prize "ICREA Acadèmia" for excellence in research, funded by the Generalitat de Catalunya. He also acknowledges the support of MOVE, where he is an affiliated researcher, and of the Barcelona Graduate School of Economics (through its Research Recognition Programme), where he is an affiliated professor. His work is also supported by the Spanish Ministry of Science and Innovation through grants ECO2008-04756 (Grupo ConsolidadoC) and CONSOLIDER-INGENIO 2010 (CDS2006-00016), and by the Generalitat de 
Catalunya through grant SGR2009-419. Inés Moreno de Barreda acknowledges financial support from the Fundación Ramón Areces.

\section{$7 \quad$ References}

Ballester, M.A., Haeringer, G., 2010. A characterization of the single-peaked domain. Soc. Choice Welfare, in press.

Barberà, S., 2001. An introduction to strategy-proof social choice functions. Soc. Choice Welfare 18, 619-653.

Barberà, S., 2010. Strategy proof social choice, in: Arrow, K., Sen, A., Suzumura, K. (Eds.), Handbook of Social Choice and Welfare, Volume 2. Kluwer Academic Publishers, North-Holland, Amsterdam, Chapter 25, pp. 731-832.

Barberà, S., Berga, D., Moreno, B., 2010. Individual versus group strategy-proofness: when do they coincide?. J. Econ. Theory 145, 1648-1674.

Barberà, S., Jackson, M., 1994. A characterization of strategy-proof social choice functions for economies with pure public goods. Soc. Choice Welfare 11, 241-252.

Barberà, S., Massó, J., Neme, A., 1997. Voting under constraints. J. Econ. Theory 76, 298-321.

Barberà, S., Massó, J., Neme, A., 2005. Voting by committees under constraints. J. Econ. Theory 122, 185-205.

Barberà, S., Massó, J., Serizawa, S., 1998. Strategy-proof voting on compact ranges. Games Econ. Behav. 25, 272-291.

Barberà, S., Sonnenschein, H., Zhou, L., 1991. Voting by committees. Econometrica 59, 595-609.

Berga, D., Serizawa, S., 2000. Maximal domain for strategy-proof rules with one public good. J. Econ. Theory 90, 39-61.

Black, D., 1948. On the rationale of group decision making. J. Polit. Econ. 56, 23-34.

Border, K.C., Jordan, J.S., 1983. Straightforward elections, unanimity and phantom voters. Rev. Econ. Stud. 50, 153-170.

Ching, S., 1997. Strategy-proofness and 'median voters'. Int. J. Game Theory 26, 473-490.

Ehlers, L., 2002. Resource-monotonic allocation when preferences are single-peaked. Econ. Theory 20, 113-131. 
Froda, A., 1929. Sur la distribution des propriétés de voisinage des fonctions de variables réelles. Thèse, Hermann, Paris.

Kar, A., Kibris, O., 2008. Allocating multiple states among agents with single-peaked preferences. Soc. Choice Welfare 31, 641-666.

Klaus, B., Bochet, O., 2010. The relation between monotonicity and strategy-proofness. Cahier de recherches économiques de DEEP No 10.01.

Klaus, K., Peters, H., Storken, T., 1998. Strategy-proof division with single-peaked preferences and individual endowments. Soc. Choice Welfare 15, 297-311.

Krehbiel, K., 2006. Pivots, in: Weingast, B.B., Wittman, D. (Eds.), The Oxford Handbook of Political Economy. Oxford University Press, Chapter 12, pp. 223-240.

McKelvey, R.D., Ordeshook, P.C., 1993. Information and elections: retrospective voting and rational expectations, in: Kinder, D.R., Palfrey, T.R. (Eds.), Experimental Foundations of Political Science. Michigan Studies in Political Analysis, pp. 333-362.

Moulin, H., 1980. On strategy-proofness and single peakedness. Public Choice 35, 437455 .

Nehring, K., Puppe, C., 2007a. The structure of strategy-proof social choice-Part I: General characterization and possibility results on median spaces. J. Econ. Theory 135, 269-305.

Nehring, K., Puppe, C., 2007b. Efficient and strategy-proof voting rules: A characterization. Games Econ. Behav. 59, 132-153.

Nisan, N., 2007. Introduction to mechanism design (for computer scientists), in: Nisan, N., Roughgarden, T., Tardos, E., Vazirani, V.V. (Eds.). Algorithmic Game Theory. Cambridge University Press, New York, pp. 209-242.

Peters, H., van der Stel, H., Storken, T., 1992. Pareto optimality, anonymity, and strategy-proofness in location problems. Int. J. Game Theory 21, 221-235.

Sprumont, Y., 1995. Strategy-proof collective choice in economic and political environments. Can. J. Econ. 28, 68-107.

Zhou, L., 1991. Impossibility of strategy-proof mechanisms for economies with pure public goods. Rev. Econ. Stud. 58, 107-119. 